\title{
Optimistic Fair Priced Oblivious Transfer
}

\author{
Alfredo Rial and Bart Preneel \\ IBBT and Katholieke Universiteit Leuven, ESAT/COSIC \\ Kasteelpark Arenberg 10, B-3001 Leuven-Heverlee, Belgium \\ firstname.lastname@esat.kuleuven.be
}

\begin{abstract}
Priced oblivious transfer (POT) is a two-party protocol between a vendor and a buyer in which the buyer purchases digital goods without the vendor learning what is bought. Although privacy properties are guaranteed, current schemes do not offer fair exchange. A malicious vendor can, e.g., prevent the buyer from retrieving the goods after receiving the payment, and a malicious buyer can also accuse an honest vendor of misbehavior without the vendor being able to prove this untrue. In order to address these problems, we define the concept of optimistic fair priced oblivious transfer and propose a generic construction that extends secure POT schemes to realize this functionality. Our construction, based on verifiably encrypted signatures, employs a neutral adjudicator that is only involved in case of dispute, and shows that disputes can be resolved without the buyer losing her privacy, i.e., the buyer does not need to disclose which digital goods she is interested in. We show that our construction can be instantiated with an existing universally composable POT scheme, and furthermore we propose a novel full-simulation secure POT scheme that is much more efficient.
\end{abstract}

Keywords: Priced oblivious transfer, verifiably encrypted signatures, fair exchange.

\section{Introduction}

The protection of privacy in e-commerce is necessary both from a marketing perspective, since privacy concerns discourage buyers from online purchasing $[1,2]$, as well as from a legal perspective, since different authorities have promulgated regulations to enforce the fulfillment of privacy policies and the confidentiality of buyer's personal data [3]. Additionally, buyers, feeling themselves in an unfavorable position at the payment phase, worry that malicious vendors can, e.g., deliver inappropriate or defective goods later on, and thus e-commerce protocols are normally analyzed in order to prove their fairness [4].

Priced oblivious transfer (POT) is a two-party protocol that provides privacy in e-commerce of digital goods by hiding from the vendor which items are bought. More formally, a vendor $\mathcal{V}$ sells a set of messages $m_{1}, \ldots, m_{N}$ with prices $p_{1}, \ldots, p_{N}$ to a buyer $\mathcal{B}$. At each purchase, $\mathcal{B}$ chooses $\tau \in\{1, \ldots, N\}$, gets $m_{\tau}$ and pays $p_{\tau}$ without $\mathcal{V}$ learning any information. 
Existing POT schemes [5-7] employ a prepaid mechanism where, initially, $\mathcal{B}$ makes a deposit, and, at each purchase, subtracts $p_{\tau}$ from the deposit with $\mathcal{V}$ learning neither $p_{\tau}$ nor the new value of the deposit. Therefore, $\mathcal{B}$ has to trust that $\mathcal{V}$, after receiving the payment, will deliver the requested goods. Furthermore, a malicious $\mathcal{V}$ can claim that $\mathcal{B}$ ran out of funds, and it is impossible for $\mathcal{B}$ to prove this untrue. On the other hand, a malicious $\mathcal{B}$ can claim that $\mathcal{V}$ is not fulfilling his delivery obligations or that her current funds are larger, and again $\mathcal{V}$ cannot prove the contrary. We propose the concept of optimistic fair POT (OFPOT) as a countermeasure.

Previous Work. Fair e-commerce is often seen as a particular case of fair exchange. Early work on fair exchange $[8,9]$ follows the approach of dividing the items to be exchanged into small pieces and exchanging them piece by piece. As noted in [10], the resulting protocol is unfair in the exchange of the last piece.

More recent work proposes the involvement of a neutral third party [11], which can be split up into several entities to avoid dependence on the reliability of a single entity [12]. When the third party is only involved in case of dispute, the protocol is called optimistic. Some of these protocols are based on verifiably encrypted signatures [13-15]. In a nutshell, $\mathcal{B}$ sends $\mathcal{V}$ a verifiably encrypted signature in her request, and, after $\mathcal{V}$ fulfills his delivery obligations, reveals a valid signature. If $\mathcal{B}$ does not reveal the signature, $\mathcal{V}$ sends the verifiably encrypted signature to the third party, which returns a valid signature after $\mathcal{V}$ demonstrates that he has fulfilled his obligations.

To the best of our knowledge, there were no previous attempts to design a fair e-commerce protocol based on POT. Existing privacy-preserving fair ecommerce protocols [16] provide buyers with anonymity, i.e., they hide from vendors the identity of buyers. As noted in [5,7], anonymous purchase has some disadvantages, like hindering customer management or making the use of currently deployed online payment methods impossible.

Our Contribution. We define an ideal functionality for OFPOT and we propose a construction that, taking a secure POT scheme as a building block, turns it into an OFPOT scheme. Our definition and construction involve a neutral third party, an adjudicator $\mathcal{A}$, which is only active in case of dispute.

Our construction is based on the use of verifiably encrypted signatures and, to some extent, resembles non-privacy preserving fair e-commerce protocols based on them. Nevertheless, we note that, since in these protocols privacy is not protected, it is trivial for $\mathcal{V}$ to show to $\mathcal{A}$ that he has fulfilled his obligations, and for $\mathcal{A}$ to verify this fact, because $\mathcal{B}$ discloses which item she requests. One of the main contributions of our work is to show that $\mathcal{A}$ can handle complaints from $\mathcal{V}$ and $\mathcal{B}$ by learning neither the list of items offered by $\mathcal{V}$ nor which ones are requested by $\mathcal{B}$.

To be proven full-simulation secure, our construction has to be instantiated with a full-simulation secure POT scheme. We show that the universally composable scheme in [7] is suitable for such an instantiation. However, the schemes in $[5,6]$ are only half-simulation secure (vendor's security definition follows the 
ideal-world/real-world paradigm, but buyer's security definition is an indistinguishability argument [17]), and thus they are not suitable. Since the scheme in [7] is rather inefficient, we propose a novel and efficient full-simulation secure POT scheme based on the oblivious transfer scheme in [18].

Outline of the Paper. In Section 2 we recall the ideal functionality for POT given in [7] and we describe our novel POT scheme. In Section 3 we define an ideal functionality for OFPOT and we depict our OFPOT scheme. Finally, Section 4 draws a conclusion and discusses future work.

\section{Efficient Priced Oblivious Transfer}

\subsection{Technical Preliminaries}

A function $\nu$ is negligible if, for every integer $c$, there exists an integer $K$ such that for all $k>K,|\nu(k)|<1 / k^{c}$. A problem is said to be hard (or intractable) if there exists no probabilistic polynomial time (p.p.t.) algorithm that solves it with non-negligible probability (in the size of the input or the security parameter).

Bilinear maps. Let $\mathbb{G}$ and $\mathbb{G}_{t}$ be groups of prime order $p$. A map $e: \mathbb{G} \times \mathbb{G} \rightarrow \mathbb{G}_{t}$ must satisfy bilinearity, i.e., $e\left(g^{x}, g^{y}\right)=e(g, g)^{x y}$; non-degeneracy, i.e., for all generators $g \in \mathbb{G}, e(g, g)$ generates $\mathbb{G}_{t} ;$ and efficiency, i.e., there exists an efficient algorithm BMGen $\left(1^{\kappa}\right)$ that outputs the pairing group setup $\left(p, \mathbb{G}, \mathbb{G}_{t}, e, g\right)$ and an efficient algorithm to compute $e(a, b)$ for any $a, b \in \mathbb{G}$.

Security Assumptions. Let $\left(p, \mathbb{G}, \mathbb{G}_{t}, e, g\right)$ be a pairing group setup. The Strong Diffie-Hellman assumption (l-SDH) [19] states that, on input $\left(g, g^{x}, \ldots\right.$, $\left.g^{x^{l}}\right) \in \mathbb{G}^{l+1}$ for $x \leftarrow \mathbb{Z}_{p}$, it is hard to output a pair $\left(c, g^{1 /(x+c)}\right)$ for $c \leftarrow \mathbb{Z}_{p}$. The Power Decisional Diffie-Hellman assumption (l-PDDH) [18] states that, on input $\left(g, g^{x}, \ldots, g^{x^{l}}, H\right) \in \mathbb{G}^{l+1} \times \mathbb{G}_{t}$ for random $H$ and $x \leftarrow \mathbb{Z}_{p}$, it is hard to distinguish between the vector $\left(H^{x}, H^{x^{2}}, \ldots, H^{x^{l}}\right) \in \mathbb{G}_{t}^{l}$ and a random vector $T \in \mathbb{G}_{t}^{l}$. As shown in [20], the $l$-PDDH assumption is implied by the $l$-BDHE assumption [21].

Signature Schemes. A signature scheme consists of the algorithms $(\mathrm{Kg}$, Sign, $\mathrm{Vf}) . \mathrm{Kg}\left(1^{\kappa}\right)$ outputs a key pair $(s k, p k)$. Sign $(s k, m)$ outputs a signature $\sigma$ on message $m . \operatorname{Vf}(p k, \sigma, m)$ outputs accept if $\sigma$ is a valid signature on $m$ and reject otherwise. A signature scheme must be correct and unforgeable [22]. Informally speaking, correctness implies that Vf always accepts a valid signature. Unforgeability means that no p.p.t adversary should be able to output a message-signature pair $(\sigma, m)$ unless he has previously obtained a signature on $m$.

We employ the weakly secure signature scheme in [19], which utilizes the SDH assumption. This scheme is existentially unforgeable under a weak chosen message attack, where the adversary submits all signature queries before seeing 
the public key. The setup consists of the pairing group setup $\left(p, \mathbb{G}, \mathbb{G}_{t}, e, g\right)$. WKg $\left(1^{\kappa}\right)$ picks random $x \leftarrow \mathbb{Z}_{p}$ and outputs a key pair $(s k, p k)=\left(x, g^{x}\right)$. WSign $(s k, m)$ computes $\sigma=g^{1 /(x+m)} . \operatorname{WVf}(p k, \sigma, m)$ outputs accept if $e(\sigma, p k$. $\left.g^{m}\right)=e(g, g)$.

Commitment schemes. A non-interactive commitment scheme consists of the algorithms (ComS, Com, Open). ComS $\left(1^{\kappa}\right)$ generates the parameters of the commitment scheme $p r_{c o m}$. Com $\left(\right.$ par $_{\text {com }}, x$,open $)$ outputs a commitment $C$ to $x$ using auxiliary information open. A commitment is opened by revealing $(x$, open $)$ and checking whether Open $\left(\right.$ par $_{\text {com }}, C, x$, open $)$ outputs accept. A commitment scheme has a hiding property and a binding property. Informally speaking, the hiding property ensures that a commitment $C$ to $x$ does not reveal any information about $x$, whereas the binding property ensures that $C$ cannot be opened to another value $x^{\prime}$. When it is clear from the context, we omit the commitment parameters par $_{\text {com }}$.

We employ the commitment scheme proposed by Pedersen [23]. PEComS $\left(1^{\kappa}\right)$ picks random generators $g, h$ of a group $\mathbb{G}$ of prime order $p$ and outputs $p a r_{\text {com }}=$ $(g, h) . \operatorname{PECom}\left(\right.$ par $_{\text {com }}, x$, open $\left._{x}\right)$ outputs $C=g^{x} h^{\text {open }} x$ on input $x \in \mathbb{Z}_{p}$ and randomness open $x \in \mathbb{Z}_{p}$. PEOpen $\left(\right.$ par $_{\text {com }}, C, x^{\prime}$, open $\left._{x}^{\prime}\right)$ outputs accept if $C=$ $g^{x^{\prime}} h^{o p e n_{x}^{\prime}}$. This scheme is information theoretically hiding and computationally binding under the discrete logarithm assumption.

Proofs of Knowledge. A zero-knowledge proof of knowledge [24] is a twoparty protocol between a prover and a verifier. The prover proves to the verifier knowledge of some secret input that fulfills some statement without disclosing this input to the verifier. The protocol should fulfill two properties. First, it should be a proof of knowledge, i.e., a prover without knowledge of the secret input convinces the verifier with negligible probability. More technically, there exists a knowledge extractor that extracts the secret input from a successful prover with all but negligible probability. Second, it should be zero-knowledge, i.e., the verifier does not learn any information about the secret input. More technically, for all possible verifiers there exists a simulator that, without knowledge of the secret input, yields a distribution that cannot be distinguished from the interaction with a real prover.

We use several existing results to prove statements about discrete logarithms: (1) proof of knowledge of a discrete logarithm modulo a prime [25]; (2) proof of knowledge of the equality of some element in different representations [26]; (3) proof of knowledge that a value $\alpha$ lies in a given interval $[0, A)[27]$; and (4) proof of the disjunction or conjunction of any two of the previous [28]. These results are often given in the form of $\Sigma$-protocols but they can be turned into zero-knowledge protocols using efficient zero-knowledge compilers [29,30], and they can be turned into non-interactive proofs in the random oracle model via the Fiat-Shamir heuristic [31].

When referring to the proofs above, we follow the notation introduced by Camenisch and Stadler [32] for various proofs of knowledge of discrete log- 
arithms and proofs of the validity of statements about discrete logarithms. $\operatorname{NIPK}\left\{(\alpha, \beta, \delta): y=g^{\alpha} h^{\beta} \wedge \tilde{y}=\tilde{g}^{\alpha} \tilde{h}^{\delta} \wedge A \leq \alpha \leq B\right\}$ denotes a "non-interactive zero-knowledge proof of knowledge of integers $\alpha, \beta$, and $\delta$ such that $y=g^{\alpha} h^{\beta}$, $\tilde{y}=\tilde{g}^{\alpha} \tilde{h}^{\delta}$ and $A \leq \alpha \leq B$ holds", where $y, g, h, \tilde{y}, \tilde{g}$, and $\tilde{h}$ are elements of some groups $G=\langle g\rangle=\langle h\rangle$ and $\tilde{G}=\langle\tilde{g}\rangle=\langle\tilde{h}\rangle$ that have the same order. (Note that some elements in the representation of $y$ and $\tilde{y}$ are equal.) The convention is that letters in the parenthesis, in this example $\alpha, \beta$, and $\delta$, denote quantities whose knowledge is being proven, while all other values are known to the verifier.

We employ the range proof proposed in [27] to prove that a value $\alpha$ lies in an interval $\left[0, d^{a}\right)$. The proof has an initialization phase where the verifier provides the prover with signatures that allow the prover to write the value $\alpha$ in base- $d$. First, the verifier runs $\operatorname{Init} \operatorname{Ver}\left(1^{\kappa}, D_{\max }\right)$, which runs $\operatorname{WKg}\left(1^{\kappa}\right)$ to get a key pair $(s k, p k)$ and computes signatures $A_{i}=\mathrm{WSign}(s k, i)$ on $d$-ary digits, i.e., $i \in \mathbb{Z}_{d}$. It also runs PEComS $\left(1^{\kappa}\right)$ to get $\operatorname{par}_{\text {com }}$ and sets par $_{\text {ran }}=$ $\left(p k,\left\{A_{i}\right\}_{i \in \mathbb{Z}_{d}}\right.$, par $\left._{\text {com }}\right)$. The verifier sends par $_{\text {ran }}$ to the prover. The prover runs $\operatorname{InitP}\left(\right.$ par $\left._{\text {ran }}\right)$ to verify the signatures by running, for $i \in \mathbb{Z}_{d}, \operatorname{WVf}\left(p k, A_{i}, i\right)$. After this initialization phase, prover and verifier can run multiple proofs. The verifier receives as input a commitment $C$, and the prover values $\left(\alpha\right.$, open $\left._{\alpha}\right)$ such that $C=\operatorname{PECom}\left(\right.$ par $_{\text {com }}, \alpha$, open $\left._{\alpha}\right)$. The prover runs $\operatorname{RProve}\left(\right.$ par $_{\text {ran }}, \alpha$, open $\left._{\alpha}\right)$, which picks $v_{j} \leftarrow \mathbb{Z}_{p}$ and computes $V_{j}=A_{\alpha_{j}}^{v_{j}}$ for every $j \in \mathbb{Z}_{a}$ such that $\alpha=\sum_{j \in \mathbb{Z}_{\alpha}} \alpha_{j} d^{j}$, and a proof pok $=\operatorname{NIPK}\left\{\left(\right.\right.$ open $\left._{\alpha},\left\{\alpha_{j}, v_{j}\right\}_{j \in \mathbb{Z}_{\alpha}}\right): C=h^{\text {open }}{ }_{\alpha}$ $\left.\prod_{j \in \mathbb{Z}_{a}}\left(g^{d^{j}}\right)^{\alpha_{j}} \wedge V_{j}=g^{v_{j} /\left(s k+\alpha_{j}\right)}\right\}$. (We abbreviate it as $\operatorname{NIPK}\left\{\left(\alpha\right.\right.$, open $\left._{\alpha}\right): 0 \leq$ $\alpha<d^{a} \wedge C=\operatorname{PEComS}\left(\right.$ par com $_{\text {com }}, \alpha$, open $\left.\left._{\alpha}\right)\right\}$.) The prover sends $\left(\left\{V_{j}\right\}_{j \in \mathbb{Z}_{a}}\right.$, pok $)$ to the verifier.

Public Key Encryption A public key encryption scheme consists of the algorithms (Kg, Enc, Dec). $\operatorname{Kg}\left(1^{\kappa}\right)$ outputs a key pair $(s k, p k)$. $\operatorname{Enc}(p k, m)$ outputs a ciphertext $c t$ on input $p k$ and a message $m$. Dec $(s k, c t)$ outputs the message $m$.

\subsection{Definition}

We define security following the ideal-world/real-world paradigm [33]. In the real world, a set of parties interact according to the protocol description in the presence of a real adversary $\mathcal{E}$, while in the ideal world dummy parties interact with an ideal functionality that carries out the desired task in the presence of an ideal adversary $\mathcal{S}$. A protocol $\psi$ is secure if there exists no environment $\mathcal{Z}$ that can distinguish whether it is interacting with adversary $\mathcal{E}$ and parties running protocol $\psi$ or with the ideal process for carrying out the desired task, where ideal adversary $\mathcal{S}$ and dummy parties interact with an ideal functionality $\mathcal{F}_{\psi}$. More formally, we say that protocol $\psi$ emulates the ideal process when, for any adversary $\mathcal{E}$, there exists a simulator $\mathcal{S}$ such that for all environments $\mathcal{Z}$, the ensembles $\operatorname{IDEAL}_{\mathcal{F}_{\psi}, \mathcal{S}, \mathcal{Z}}$ and $\mathrm{REAL}_{\psi, \mathcal{E}, \mathcal{Z}}$ are computationally indistinguishable. We refer to [33] for a description of how these ensembles are constructed.

We recall the ideal functionality $\mathcal{F}_{\text {РОт }}$ for priced oblivious transfer in [7]. Every functionality and every protocol invocation should be instantiated with a 
unique session-ID that distinguishes it from other instantiations. For the sake of ease of notation, we omit session-IDs from our description.

\section{Functionality $\mathcal{F}_{\text {POT }}$}

Parameterized with the number of messages $N$, the message length $l$, the maximum price $p_{\max }$, and the maximum deposit $D_{\max }$, and running with a vendor $\mathcal{V}$ and a buyer $\mathcal{B}, \mathcal{F}_{\text {POT }}$ works as follows:

- On input a message (init, $\left.m_{1}, p_{1}, \ldots, m_{N}, p_{N}\right)$ from $\mathcal{V}$, where each $m_{i} \in$ $\{0,1\}^{l}$ and each $p_{i} \in\left[0, p_{\max }\right]$, it stores $\left(m_{1}, p_{1}, \ldots, m_{N}, p_{N}\right)$ and sends (init, $p_{1}, \ldots, p_{N}$ ) to $\mathcal{B}$.

- On input a message (deposit, $a c$ ), where $a c \in\left[0, D_{\max }\right.$ ), if a (init,...) message was not received before, then it does nothing. Otherwise, it stores $a c$ and sends (deposit, $a c$ ) to $\mathcal{V}$.

- On input a message (request, $\tau$ ) from $\mathcal{B}$, where $\tau \in\{1, \ldots, N\}$, if either messages (init, $\left.m_{1}, p_{1}, \ldots, m_{N}, p_{N}\right)$ and (deposit, $a c$ ) were not received before or $a c-p_{\tau}<0$, then it does nothing. Otherwise, it sends (request) to $\mathcal{V}$ and receives (response, $b$ ) in response. If $b=0$, it sends (response, $\perp$ ) to $\mathcal{B}$. If $b=1$, it updates $a c=a c-p_{\tau}$ and sends (response, $m_{\tau}$ ) to $\mathcal{B}$.

\subsection{Intuition Behind Our Construction}

Our POT scheme is based on the adaptive oblivious transfer scheme in [18]. It is an assisted decryption scheme in which there is an initialization phase and several purchase phases. At the initialization phase, $\mathcal{V}$ sends $\mathcal{B}$ a collection of ciphertexts that encrypt the messages $m_{1}, \ldots, m_{N}$ to be sold, and at each purchase $\mathcal{V}$ helps $\mathcal{B}$ to decrypt one of them. As noted in [34], this design approach leads to purchase phases with constant communication and computation complexity, and ensures that $\mathcal{V}$ cannot modify the messages after the initialization phase.

Each ciphertext $C_{i}$ consists of a unique price $^{1} p_{i}$, a signature on the price $A_{i}$ and an encryption $B_{i}=e\left(h, A_{i}\right) \cdot m_{i}$, where $h$ is the secret key of $\mathcal{V}$. To compute a request for $m_{\tau}, \mathcal{B}$ sends $\mathcal{V}$ a blinded value $V=A_{\tau}^{v}$ together with a zero-knowledge proof that $\mathcal{B}$ possesses a valid signature $A_{\tau}$ and that $V$ is correctly computed. $\mathcal{V}$ computes a blinded decryption $W=e(h, V)$ and proves that the secret key $h$ was used to compute $W$. Finally, $\mathcal{B}$ unblinds $W$ to decrypt the message $m_{\tau}$ via $B_{\tau} /\left(W^{1 / v}\right)$.

To allow for oblivious payments, our POT scheme follows the prepaid mechanism by [7]. At the initialization phase, $\mathcal{B}$ makes a deposit $a c_{0}$ and sends a commitment $D_{0}$ to the deposit and its opening to $\mathcal{V}$. At purchase phase $i, \mathcal{B}$

\footnotetext{
${ }^{1}$ If several messages have the same price, unique prices can be obtained by adequately scaling prices and accounts [5].
} 
sends a commitment $D_{i}$ to the new value of the account $a c_{i}$ along with a zeroknowledge proof that $a c_{i}=a c_{i-1}-p_{\tau}$ and that $a c_{i} \in\left[0, D_{\max }\right)$, to prove that the account is correctly updated and that $\mathcal{B}$ has enough funds to buy $m_{\tau}$. For the latter, we employ the range proof recently proposed in [27].

We prove that our scheme realizes $\mathcal{F}_{\text {POT }}$ under the SDH and PDDH assumptions (used in [18]), and under the assumption the binding and hiding properties of the commitment scheme hold. In the description of the scheme given below, we compute non-interactive proofs via Fiat-Shamir heuristic [31] and thus the construction is secure in the random oracle model [35]. This is convenient when using the scheme as a building block of the OFPOT scheme depicted in Section 3 , because it allows $\mathcal{A}$ to verify requests and responses non-interactively. Nevertheless, for other uses of the scheme interactive proofs of knowledge can be employed, yielding a construction in the standard model.

\subsection{Description of the Scheme}

We begin with a high level description of the POT scheme. Details on the algorithms can be found below.

\section{POT}

Initialization phase. On input (init, $m_{1}, p_{1}, \ldots, m_{N}, p_{N}$ ), in which each price is unique, $\mathcal{V}$ runs $\operatorname{POTInitV}\left(1^{\kappa}, m_{1}, p_{1}, \ldots, m_{N}, p_{N}, D_{\max }\right)$ in order to obtain a database commitment $T$ and a key pair $\left(p k_{\mathcal{V}}, s k_{\mathcal{V}}\right)$, and sends $\left(p k_{\mathcal{V}}, T\right)$ to $\mathcal{B}$. On input (deposit, $\left.a c_{0}\right), \mathcal{B}$ runs $\left(P, D_{0}^{\prime}\right) \leftarrow$ POTInitB $\left(1^{\kappa}, p k_{\mathcal{V}}, T, a c_{0}\right)$ and aborts if the output is reject. Otherwise, $\mathcal{B}$ sends the payment message $(P)$ to $\mathcal{V}$ and pays an amount of $a c_{0}$ through an arbitrary payment channel. $\mathcal{V}$ runs $\left(D_{0}, a c_{0}\right) \leftarrow$ POTGetDep $\left(s k_{\mathcal{V}}, P, D_{\max }\right)$ and checks that $a c_{0}$ corresponds to the amount of money received. $\mathcal{V}$ stores state information $V_{0}=\left(T, s k_{\mathcal{V}}\right.$, $\left.p k_{\mathcal{V}}, D_{0}\right)$ and outputs (deposit, $a c_{0}$ ), and $\mathcal{B}$ stores state information $B_{0}=\left(p k_{\mathcal{V}}, T, D_{0}^{\prime}\right)$.

Transfer phase. On input (request, $\left.\tau_{i}\right), \mathcal{B}$ runs POTReq $\left(p k_{\mathcal{V}}, T, D_{i-1}^{\prime}, \tau_{i}\right)$ to get a request $Q$ and private state $\left(Q^{\prime}, D_{i}^{\prime}\right)$. $\mathcal{B}$ sends $(Q)$ and stores $\left(Q^{\prime}, D_{i}^{\prime}\right)$. On input (response, $b$ ), if $b=0 \mathcal{V}$ sends $(\perp)$ to $\mathcal{B}$. If $b=1$, $\mathcal{V}$ runs $\operatorname{POTVerReq}\left(p k_{\mathcal{V}}, D_{i-1}, Q\right)$ and ignores the request if the output is reject. Otherwise $\mathcal{V}$ runs $\operatorname{POTResp}\left(p k_{\mathcal{V}}, s k_{\mathcal{V}}, Q\right)$ to obtain a response $R$ and state $D_{i}$, and sends $(R)$ to $\mathcal{B}$. $\mathcal{B}$ runs POTVerResp $\left(p k_{\mathcal{V}}, R\right)$ and outputs (response, $\perp$ ) it the output is reject. Otherwise $\mathcal{B}$ runs POTComplete $\left(T, R, Q^{\prime}\right)$ to obtain $m_{\tau_{i}}$. $\mathcal{V}$ stores state information $V_{i}=$ $\left(s k_{\mathcal{V}}, T, p k_{\mathcal{V}}, D_{i}\right)$, and $\mathcal{B}$ stores state information $B_{i}=\left(T, p k_{\mathcal{V}}, D_{i}^{\prime}\right)$ and outputs (response, $m_{\tau_{i}}$ ). 
POTInitV $\left(1^{\kappa}, m_{1}, p_{1}, \ldots, m_{N}, p_{N}, D_{\max }\right)$ computes a pairing group $\operatorname{setup} \Phi=$ $\left(p, \mathbb{G}, \mathbb{G}_{t}, e, g\right)$, picks a random generator $h \in \mathbb{G}$ and sets $H=e(g, h)$. It runs InitVer $\left(1^{\kappa}, D_{\max }\right.$ ) to obtain $\operatorname{par}_{\text {ran }}$ (which includes parameters of the commitment scheme $\left.\operatorname{par}_{c o m}\right)$ and $\mathrm{Kg}\left(1^{\kappa}\right)$ to obtain $\left(s k_{e n c}, p k_{e n c}\right) .^{2}$ It also runs $\operatorname{WKg}\left(1^{\kappa}\right)$ to obtain a key pair $(s k, p k)$ for the signature scheme, and, for $i=1$ to $N$, it computes $A_{i}=\mathrm{WSign}\left(s k, p_{i}\right)$ and $B_{i}=e\left(h, A_{i}\right) \cdot m_{i}$. It sets $C_{i}=\left(A_{i}, B_{i}, p_{i}\right)$. It computes a non-interactive proof $\pi_{1}=\operatorname{NIPK}\{(h): H=$ $e(g, h)\}$. It outputs $s k_{\mathcal{V}}=\left(s k_{e n c}, h\right), p k_{\mathcal{V}}=\left(\Phi, H, p a r_{\text {ran }}, p k, p k_{\text {enc }}, \pi_{1}\right)$ and $T=\left(C_{1}, \ldots, C_{N}\right)$.

POTInitB $\left(1^{\kappa}, p k_{\mathcal{V}}, T, a c_{0}\right)$ parses, for $i=1$ to $N, C_{i}$ as $\left(A_{i}, B_{i}, p_{i}\right)$, and checks whether $\mathrm{WVf}\left(p k, A_{i}, p_{i}\right)$ outputs accept. It also verifies $\pi_{1}$ and checks $p a r_{\text {ran }}$ via InitP $\left(\right.$ par $\left._{r a n}\right)$. Then it runs $\operatorname{Enc}\left(p k_{e n c}, a c_{0}\right)$ to obtain an encryption $c t$ and computes a commitment $D_{0}=\operatorname{PECom}\left(\right.$ par $\left._{c o m}, a c_{0}, o p e n_{a c_{0}}\right)$ for random open $_{a c_{0} .} \cdot{ }^{3}$ It sets $P=\left(\right.$ ct,open $\left.{ }_{a c_{0}}\right)$ and $D_{0}^{\prime}=\left(a c_{0}\right.$, open $\left._{a c_{0}}, D_{0}\right)$. It outputs $\left(P, D_{0}^{\prime}\right)$.

POTGetDep $\left(s k_{\mathcal{V}}, P, D_{\max }\right)$ runs $\operatorname{Dec}\left(s k_{e n c}, c t\right)$ to obtain $a c_{0}$ and checks that $a c_{0} \in\left[0, D_{\max }\right)$. It computes $D_{0}=$ PECom $\left(\right.$ par $_{\text {com }}, a c_{0}$, open $\left._{a c_{0}}\right)$ and outputs $\left(D_{0}, a c_{0}\right)$.

$\operatorname{POTReq}\left(p k_{\mathcal{V}}, T, D_{i-1}^{\prime}, \tau\right)$ calculates $a c_{i}=a c_{i-1}-p_{\tau}$, picks random open $_{p}$, open $\left._{a c_{i}}\right) \leftarrow \mathbb{Z}_{p}$, and computes $D_{p}=\operatorname{PECom}\left(\right.$ par $_{\text {com }}, p_{\tau}$, open $\left._{p}\right)$ and $D_{i}=$ PECom $\left(\right.$ par $_{\text {com }}, a c_{i}$, open $\left._{a c_{i}}\right)$. It picks random $v \in \mathbb{Z}_{p}$ and computes $V=A_{\tau}^{v}$. It computes a non-interactive proof ${ }^{4} \pi_{2}$ :

$$
\begin{aligned}
& \operatorname{NIPK}\left\{\left(p_{\tau}, \text { open }_{p}, a c_{i}, \text { open }_{a c_{i}}, v, \alpha\right)\right. \text { : } \\
& e(V, p k)=e(V, g)^{-p_{\tau}} e(g, g)^{v} \wedge \\
& D_{p}=\operatorname{PECom}\left(\text { par }_{\text {com }}, p_{\tau}, \text { open }_{p}\right) \wedge \\
& D_{i}=\operatorname{PECom}\left(\text { par }_{\text {com }}, a c_{i}, \text { open }_{a c_{i}}\right) \wedge \\
& D_{i-1} /\left(D_{i} D_{p}\right)=\tilde{h}^{\alpha} \wedge \\
& \left.0 \leq a c_{i}<D_{\max }\right\} \text {. }
\end{aligned}
$$

Equation 1 proves that $V=A_{\tau}^{v}$ and that $A_{\tau}$ is a signature computed by $\mathcal{V}$. Equations 2 and 3 prove knowledge of the committed price $p_{\tau}$ and $a c_{i}$ respectively, where $p_{\tau}$ is the value signed in $A_{\tau}$. Equation 4 proves that $a c_{i}=$ $a c_{i-1}-p_{\tau}$, and Equation 5 proves that $a c_{i}$ is non-negative. The algorithm outputs $Q=\left(V, D_{p}, D_{i}, \pi_{2}\right), Q^{\prime}=(v, \tau)$ and $D_{i}^{\prime}=\left(a c_{i}\right.$, open $\left._{a c_{i}}, D_{i}\right)$.

POTVerReq $\left(p k_{\mathcal{V}}, D_{i-1}, Q\right)$ verifies $\pi_{2}$ and outputs either accept or reject.

POTResp $\left(p k_{\mathcal{V}}, s k_{\mathcal{V}}, Q\right)$ parses $Q$ as $\left(V, D_{p}, D_{i}, \pi_{2}\right)$ and computes $W=e(h, V)$ and a proof $\pi_{3}=\operatorname{NIPK}\{(h): H=e(g, h) \wedge W=e(V, h)\}$ that $W$ is computed correctly by using the request $V$ and the secret key $h$. It outputs $R=\left(W, \pi_{3}\right)$ and $D_{i}$.

\footnotetext{
${ }^{2}$ We can employ any IND-CPA secure encryption scheme.

${ }^{3}$ Although it would be possible to use a fixed value open ${ }_{a c_{0}}$, this allows $\mathcal{B}$ to hide her deposit from the adjudicator in the OFPOT scheme.

${ }^{4} \tilde{h}$ is included in the parameters of the commitment scheme $\operatorname{par}_{\text {com }}=(\tilde{g}, \tilde{h})$.
} 
POTVerResp $\left(p k_{\mathcal{V}}, R\right)$ verifies $\pi_{3}$ and outputs either accept or reject.

POTComplete $\left(T, R, Q^{\prime}\right)$ parses $Q^{\prime}$ as $(v, \tau), R$ as $\left(W, \pi_{3}\right)$ and $C_{\tau}$ as $\left(A_{\tau}, B_{\tau}, p_{\tau}\right)$. It outputs $m_{\tau}=B_{\tau} /\left(W^{1 / v}\right)$.

Theorem 1. This POT scheme securely realizes $\mathcal{F}_{\mathrm{POT}}$.

We prove this theorem in Appendix A. As for efficiency, we note that the communication and computation cost of our protocol is roughly that of the OT scheme in [18], plus the overhead introduced by the range proof in [27]. We refer to [34] for a comparison of the efficiency of several OT schemes, and to [27] for efficiency measurements of the range proof.

\section{An Optimistic Fair POT Scheme}

\subsection{Technical Preliminaries}

Verifiably Encrypted Signatures. A verifiably encrypted signature (VES) scheme consists of algorithms ( $\mathrm{Kg}, \mathrm{AdjKg}$, Sign, Vf, Create, VesVf, Adj). Algorithm $\mathrm{Kg}\left(1^{\kappa}\right)$ outputs a key pair $(s k, p k) . \operatorname{AdjKg}\left(1^{\kappa}\right)$ outputs a key pair $(a s k, a p k)$ for the adjudicator. $\operatorname{Sign}(s k, m)$ computes a signature $\sigma$ under $s k$ on a message $m . \operatorname{Vf}(p k, \sigma, m)$ outputs accept if $\sigma$ is a valid signature on $m$ under $p k$. Create $(s k, a p k, m)$ outputs a verifiably encrypted signature $\omega$ on input the secret key $s k$, the adjudicator's public key apk and the message $m$. $\operatorname{Ves} \vee \mathrm{f}(p k, a p k, \omega, m)$ outputs accept if $\omega$ is a valid verifiably encrypted signature on input the public key $p k$, the adjudicator's public key $a p k$ and the message $m . \operatorname{Adj}(p k, a s k, a p k, \omega$, $m$ ) outputs an ordinary signature $\sigma$ on $m$ if $\omega$ is valid.

A VES scheme is complete if, for all key pairs $(s k, p k)$ computed by $\operatorname{Kg}\left(1^{\kappa}\right)$ and for all key pairs $(a s k, a p k)$ computed by $\operatorname{AdjKg}\left(1^{\kappa}\right)$, it holds that $\operatorname{Ves} \mathrm{Vf}(p k$, $a p k$, Create $(s k, a p k, m), m)$ outputs accept and algorithm $\vee \mathrm{f}(p k, \operatorname{Adj}(p k, a s k, a p k$, Create $(s k, a p k, m), m), m)$ outputs accept.

Security for verifiably encrypted signatures $[13,15]$ is defined via four properties: unforgeability, opacity, extractability and abuse-freeness. Roughly speaking, unforgeability ensures that it is intractable to compute a verifiably encrypted signature on behalf of another user. Opacity ensures that nobody but the adjudicator and the signer can obtain an ordinary signature from a verifiably encrypted signature. Extractability means that the adjudicator is able to extract an ordinary signature from a valid verifiably encrypted signature with all but negligible probability. Abuse-freeness prevents an adversary that colludes with the adjudicator from forging verifiably encrypted signatures.

\subsection{Definition}

Previous work on defining optimistic fair exchange in the ideal-world/real-world paradigm only covers the case where buyer's privacy is not protected [36]. Therefore, we define an ideal functionality $\mathcal{F}_{\text {OFPOT }}$ for OFPOT that extends $\mathcal{F}_{\text {POT }}$. It describes the functionality provided by our construction when the adjudicator $\mathcal{A}$ 
is neutral, and we prove security under this restriction. (We explain more intuitively the functionality provided by $\mathcal{F}_{\text {ОFРОт }}$ in Section 3.3.) We also recall the description of a functionality $\mathcal{F}_{\mathrm{CRS}}$ given in [37], which is used when the POT scheme used to instantiate the OFPOT scheme operates in the $\mathcal{F}_{\mathrm{CRS}}$-hybrid model, where parties have access to an honestly-generated common reference string.

\section{Functionality $\mathcal{F}_{\mathrm{CRS}}$}

Parameterized with a distribution $D$ and a set of participants $\mathcal{P}$, on input (crs) from party $P$, if $P \notin \mathcal{P}$ it aborts. Otherwise, if there is no value $r$ recorded, it picks $r \leftarrow D$ and records $r$. It sends (crs, $r$ ) to $P$.

\section{Functionality $\mathcal{F}_{\text {OFPOT }}$}

Parameterized with $\left(N, l, p_{\max }, D_{\max }\right)$, and running with a vendor $\mathcal{V}$, a buyer $\mathcal{B}$ and an adjudicator $\mathcal{A}, \mathcal{F}_{\text {OFPOT }}$ works as follows:

- On input a message (init, $m_{1}, p_{1}, \ldots, m_{N}, p_{N}$ ) from $\mathcal{V}$, it behaves as $\mathcal{F}_{\mathrm{POT}}$.

- On input a message (deposit, $a c_{0}$ ) from $\mathcal{B}$, it behaves as $\mathcal{F}_{\text {POT. }}$.

- On input a message (request, $i, \tau)$ from $\mathcal{B}$, where $\tau \in\{1, \ldots, N\}$, if messages (init, ...) or (deposit, ...) were not received before, $i$ does not equal the number of the transfer $t, a c_{i}-p_{\tau}<0$, or dispute $_{i}=1$, then it does nothing. Otherwise, it sends (request, $i$ ) to $\mathcal{V}$ and receives (response, $i, b$ ) in response. It stores $(i, \tau, b)$. If $b=0$, it sends (response, $i, \perp$ ) to $\mathcal{B}$. If $b=1$, it sends (response, $i, m_{\tau}$ ) to $\mathcal{B} . \mathcal{B}$ returns a message (complete, $i, c$ ), which is handed to $\mathcal{V}$. If $c=1$, it sets $a c_{i}=a c_{i-1}-p_{\tau}$ and updates the number of transfers $t=t+1$. If $c=0$, it sets dispute $_{i}=1$.

- On input a message $\left(\operatorname{complain}_{\mathcal{V}}, i\right)$ from $\mathcal{V}$, if dispute $_{i}=0$ or $t \neq i$, then it sends $\left(\operatorname{compInv}_{\mathcal{V}}\right)$ to $\mathcal{V}$ and (complain $\mathcal{V}, i$,inv) to $\mathcal{A}$. Otherwise it sets $a c_{i}=a c_{i-1}-p_{\tau}$, updates the number of transfers $t=t+1$ and sends $\left(\operatorname{compSolved}_{\mathcal{V}}\right)$ to $\mathcal{V},\left(\operatorname{compResp}_{\mathcal{V}}, i, m_{\tau}\right)$ to $\mathcal{B}$ and $\left(\operatorname{complain}_{\mathcal{V}}, i\right.$, solved $)$ to $\mathcal{A}$.

- On input a message $\left(\operatorname{complain}_{\mathcal{B}}, i, \tau\right)$ from $\mathcal{B}$, if $t \neq i$ or if there exists a tuple $(i, \tau, b)$ and $\tau \neq \tau^{\prime}$, then it sends $\left(\operatorname{compInv}_{\mathcal{B}}\right)$ to $\mathcal{B}$ and $\left(\right.$ complain $_{\mathcal{B}}, i$, inv $)$ to $\mathcal{A}$. Otherwise it sends $\left(\operatorname{complain}_{\mathcal{B}}, i\right)$ to $\mathcal{V}$. Upon receiving (collaborate, $i, b$ ) from $\mathcal{V}$, if $b=0$ it sends (guilty $\mathcal{V}$ ) to $\mathcal{V}$ and $\mathcal{B}$ and $\left(\operatorname{complain}_{\mathcal{B}}, i\right.$, guilty $\left._{\mathcal{V}}\right)$ to $\mathcal{A}$. If $b=1$ it sets $a c_{i}=a c_{i-1}-p_{\tau}$, updates the number of transfers $t=t+1$, sends $\left(\operatorname{compResp}_{\mathcal{B}}, i, m_{\tau}\right)$ to $\mathcal{B},\left(\right.$ compSolved $\left._{\mathcal{B}}\right)$ to $\mathcal{V}$ and $\left(\operatorname{complain}_{\mathcal{B}}, i\right.$, solved $)$ to $\mathcal{A}$. 


\subsection{Intuition Behind Our Construction}

Our OFPOT scheme extends a full-simulation secure POT scheme with a fair exchange protocol based on verifiably encrypted signatures. In a nutshell, at a purchase phase, $\mathcal{B}$ computes her request following the POT scheme and a verifiably encrypted signature that signs the request. Then $\mathcal{V}$ computes a response following the POT scheme. If $\mathcal{B}$ is satisfied, $\mathcal{B}$ reveals a valid signature on her request, and otherwise $\mathcal{B}$ complains. $\mathcal{V}$ also complains when he computed his response honestly and $\mathcal{B}$ did not reveal a valid signature.

More concretely, the initialization phase follows that of the POT scheme. Additionally, $\mathcal{B}$ signs the payment message she sends to $\mathcal{V}, \mathcal{A}$ hands its public key to $\mathcal{V}$ and $\mathcal{B}$, and $\mathcal{A}$ receives the public keys of $\mathcal{V}$ and $\mathcal{B}$. At purchase phase $i$, $\mathcal{B}$ computes a request $Q_{i}$ following algorithm POTReq of the POT scheme, and computes a verifiably encrypted signature $\omega_{i}$ on $H\left(i, Q_{i}\right)$, where $H$ is a collisionresistant hash function. $\mathcal{V}$ rejects the request if $i$ is not the valid purchase index, i.e., if $\mathcal{V}$ did not receive a signature $\sigma_{i}$ on $H\left(i-1, Q_{i-1}\right)$ or if $\mathcal{V}$ already received a signature on $H\left(i, Q_{i}^{\prime}\right)$. Otherwise $\mathcal{V}$ computes a response $R$ following algorithm POTResp of the POT scheme. Finally, $\mathcal{B}$ obtains the message $m_{\tau}$ by running algorithm POTComplete and reveals to $\mathcal{V}$ a signature $\sigma_{i}$ on $H\left(i, Q_{i}\right) .^{5}$

When $\mathcal{B}$ does not reveal $\sigma_{i}, \mathcal{V}$ complains by sending to $\mathcal{A}$ the request $\left(i, Q_{i}, \omega_{i}\right)$ and the response $R$. $\mathcal{A}$ verifies both request and response, sends $R$ to $\mathcal{B}$, extracts $\sigma_{i}$ from $\omega_{i}$ and sends $\sigma_{i}$ to $\mathcal{V}$. Verification by $\mathcal{A}$ can be done without knowing the choice $\tau$ of $\mathcal{B}$. We note that a malicious $\mathcal{V}$ cannot produce fake requests on behalf of $\mathcal{B}$ thanks to the unforgeability property of the VES scheme. Moreover, if $\mathcal{B}$ did not reveal $\sigma_{i}$ because the response $R^{\prime}$ from $\mathcal{V}$ was not correct, after $\mathcal{V}$ complains $\mathcal{A}$ ensures that $\mathcal{B}$ receives a valid response.

When the response sent by $\mathcal{V}$ is not correct, $\mathcal{B}$ complains by sending to $\mathcal{A}$ a request $\left(i, Q_{i}, \omega_{i}\right) .{ }^{6} \mathcal{A}$ tells $\mathcal{V}$ to send $\left(j, Q_{j}, \sigma_{j}\right)$ for $j=i-1$ in order to check if the complaint is valid. (When $i=1, \mathcal{V}$ should send the signature on the payment message.) If $\mathcal{V}$ sends it for $j \geq i$, then $\mathcal{A}$ states that the complaint is invalid because this means that $\mathcal{B}$ has already shown conformity for purchase $i$, and if $j<i-1$, then it asks $\mathcal{B}$ to send a request with index $j .{ }^{7}$ If $\mathcal{V}$ does not reveal any signature, $\mathcal{A}$ finds $\mathcal{V}$ guilty. (We note that $\mathcal{V}$ cannot produce signatures on behalf of $\mathcal{B}$ thanks to the opacity property of the VES scheme.) Otherwise, $\mathcal{A}$ verifies the request from $\mathcal{B}$ and sends it to $\mathcal{V}$. If $\mathcal{V}$ returns a different tuple $\left(i, Q_{i}^{\prime}, \omega_{i}^{\prime}\right)$, then $\mathcal{A}$ states that the complaint is invalid because this may mean that a malicious $\mathcal{B}$ already obtained the message requested in $Q_{i}^{\prime}$, and is trying to obtain a different message. Otherwise, if $\mathcal{V}$ returns a valid response $R$, $\mathcal{A}$ forwards $R$ to $\mathcal{B}$ and reveals $\sigma_{i}$ to $\mathcal{V}$. If not, $\mathcal{A}$ finds $\mathcal{V}$ guilty.

\footnotetext{
${ }^{5}$ In practical implementations, this message can be sent together with the next request in order to save communication rounds.

${ }^{6}$ We note that $\mathcal{B}$ can complain without previously interacting with $\mathcal{V}$, but this does not give a malicious $\mathcal{B}$ any advantage over $\mathcal{V}$.

${ }^{7}$ We note that $\mathcal{V}$ should always send the larger index $j$ for which he possesses a signature, because otherwise he would be claiming that some purchases did not happen, thus increasing the account of $\mathcal{B}$.
} 
We prove that our scheme realizes $\mathcal{F}_{\text {OFPOT }}$ if it is instantiated with a fullsimulation secure POT scheme (which realizes $\mathcal{F}_{\mathrm{POT}}$ ) and under the assumptions that the VES scheme is unforgeable, opaque and extractable, and that $\mathcal{A}$ is neutral. The abuse-freeness property of the VES scheme gives $\mathcal{B}$ some protection when $\mathcal{V}$ and $\mathcal{A}$ collude: they cannot produce fake requests. However, once $\mathcal{B}$ sends a request, a malicious $\mathcal{A}$ can reveal $\sigma_{i}$ to $\mathcal{V}$ without sending $R$ to $\mathcal{B}$. (Nevertheless, privacy of $\mathcal{B}$ is still preserved.) Similarly, when $\mathcal{B}$ and $\mathcal{A}$ collude, $\mathcal{V}$ is not protected.

When our construction is instantiated with the universally composable POT scheme in [7] (which we summarize in Appendix C), we obtain a UC secure construction in the common reference string model. (In the description given below, we include the common reference string as input of the algorithms, but it should be removed when unnecessary.) When instantiated with the POT scheme proposed in Section 2, we obtain a full-simulation secure and more efficient scheme.

Finally, we point out that our scheme does not ensure that all the buyers receive the same messages, or that the messages that $\mathcal{V}$ sells fulfill the expectations of $\mathcal{B}$. Nevertheless, there already exist countermeasures which employ a third party against these problems [38], and $\mathcal{A}$ can apply them. Basically, these countermeasures ensure that all the buyers obtain the same messages from $\mathcal{V}$. We can achieve this by ensuring that they obtain the same database commitment $T$.

\subsection{Description of the Scheme}

We begin with a high level description of the OFPOT scheme. Details on the algorithms can be found below. We recall that the scheme is parameterized with $\left(N, l, p_{\max }, D_{\max }\right)$.

\section{OFPOT}

Initialization phase. On input (init, $\left.m_{1}, p_{1}, \ldots, m_{N}, p_{N}\right), \mathcal{V}$ queries $\mathcal{F}_{\mathrm{CRS}}$ with (crs), which runs POTGenCRS $\left(1^{\kappa}, p_{\max }, D_{\max }\right)$ and returns (crs, crs $). \mathcal{B}$ and $\mathcal{A}$ also query $\mathcal{F}_{\mathrm{CRS}}$ with (crs), which returns (crs, crs). $\mathcal{V}$ runs POTInitV $\left(\right.$ crs $\left., m_{1}, p_{1}, \ldots, m_{N}, p_{N}, D_{\max }\right)$ to get a database commitment $T$ and a key pair $\left(s k_{\mathcal{V}}, p k_{\mathcal{V}}\right)$, and sends $\left(p k_{\mathcal{V}}, T\right)$ to $\mathcal{B}$. On input (deposit, $\left.a c_{0}\right), \mathcal{B}$ computes $\left(P, D_{0}^{\prime}\right) \leftarrow \operatorname{POTInitB}\left(c r s, p k_{\mathcal{V}}, T, a c_{0}\right)$ to obtain a payment message $P$ and the opening of the commitment to the account $D_{0}^{\prime}$, and aborts if the output is reject. Otherwise, $\mathcal{B}$ runs $\left(s k_{\mathcal{B}}, p k_{\mathcal{B}}, \sigma_{0}\right) \leftarrow \mathrm{OFInitB}\left(c r s, D_{0}^{\prime}\right)$ to obtain a key pair $\left(s k_{\mathcal{B}}, p k_{\mathcal{B}}\right)$ and a signature $\sigma_{0}$ on $D_{0}$, sends $\left(P, p k_{\mathcal{B}}, \sigma_{0}\right)$ and pays $a c_{0}$ to $\mathcal{V}$ through an arbitrary payment channel. $\mathcal{V}$ runs $\left(D_{0}, a c_{0}\right) \leftarrow$ POTGetDep $\left(c r s, s k_{\mathcal{V}}, P, D_{\max }\right)$ to obtain the commitment $D_{0}$ and checks that $a c_{0}$ equals the amount of money paid. $\mathcal{V}$ runs $\operatorname{OFInitV}\left(p k_{\mathcal{B}}, \sigma_{0}, D_{0}\right)$ 
to check $\sigma_{0}$ and aborts if the output is reject. $\mathcal{A}$ runs $(a s k, a p k) \leftarrow$ OFInitA $($ crs $)$ and sends apk to $\mathcal{V}$ and $\mathcal{B}$. $\mathcal{V}$ and $\mathcal{B}$ retrieve apk. $\mathcal{V}(\mathcal{B})$ sends $\left(p k_{\mathcal{V}}, p k_{\mathcal{B}}\right)\left(\left(p k_{\mathcal{V}}^{\prime}, p k_{\mathcal{B}}^{\prime}\right)\right)$ to $\mathcal{A}, \mathcal{A}$ checks equality of the public keys and stores $\left(p k_{\mathcal{V}}, p k_{\mathcal{B}}, a s k\right)$. (Alternatively, $\mathcal{A}, \mathcal{V}$ and $\mathcal{B}$ can use a PKI.) $\mathcal{V}$ stores state information $V_{0}=\left(s k_{\mathcal{V}}, T, p k_{\mathcal{V}}, p k_{\mathcal{B}}, a p k, D_{0}, \sigma_{0}\right)$ and outputs (deposit, $a c_{0}$ ), and $\mathcal{B}$ stores state information $B_{0}=$ $\left(s k_{\mathcal{B}}, T, p k_{\mathcal{B}}, p k_{\mathcal{V}}, a p k, D_{0}^{\prime}\right)$ and outputs (init, $\left.p_{1}, \ldots, p_{N}\right)$.

Transfer phase. On input (request, $i, \tau), \mathcal{B}$ does nothing if $\mathcal{B}$ has previously received (request, $i, \ldots)$. Otherwise $\mathcal{B}$ runs $\left(Q_{i}, Q_{i}^{\prime}, D_{i}^{\prime}\right) \leftarrow$ POTReq $\left(c r s, p k_{\mathcal{V}}, T, D_{i-1}^{\prime}, \tau\right)$ to compute a request message $Q_{i}$, trapdoor information $Q_{i}^{\prime}$ and opening $D_{i}^{\prime}$, and $\omega_{i} \leftarrow \operatorname{OFReq}\left(s k_{\mathcal{B}}, a p k, i, Q_{i}\right)$ to obtain a verifiably encrypted signature $\omega_{i}$ on $Q_{i}$. $\mathcal{B}$ sends the request $\left(i, Q_{i}, \omega_{i}\right)$ and stores $\left(Q_{i}^{\prime}, D_{i}^{\prime}\right)$. Upon receiving $\left(i, Q_{i}, \omega_{i}\right)$, if $i$ does not equal the number of the transfer $t, \mathcal{V}$ does nothing. Otherwise, on input (response, $b$ ), if $b=0 \mathcal{V}$ sends $(\perp)$ to $\mathcal{B}$. If $b=1 \mathcal{V}$ runs POTVerReq $\left(c r s, p k_{\mathcal{V}}, D_{i-1}, Q_{i}\right)$ and $\operatorname{OFVerReq}\left(p k_{\mathcal{B}}, a p k, \omega_{i}, i, Q_{i}\right)$. If any of the two algorithms outputs reject, $\mathcal{V}$ rejects the request. Otherwise $\mathcal{V}$ runs $(R) \leftarrow \operatorname{POTResp}\left(c r s, p k_{\mathcal{V}}, s k_{\mathcal{V}}, Q_{i}\right)$, sends the response $(R)$ and keeps state $\left(i, Q_{i}, \omega_{i}\right)$. If the output of POTVerResp $\left(c r s, p k_{\mathcal{V}}, R\right)$ is reject, $\mathcal{B}$ outputs (response, $i, \perp$ ) and proceeds with the complaint phase. Otherwise, $\mathcal{B}$ runs $\left(m_{\tau}\right) \leftarrow$ POTComplete $\left(\mathrm{crs}, T, R, Q_{i}^{\prime}\right)$ and outputs (response, $i, m_{\tau}$ ). On input (complete, $c, i$ ), if $c=0, \mathcal{B}$ sends $(\perp)$, and, if $c=1, \mathcal{B}$ runs $\sigma_{i} \leftarrow \operatorname{OFComplete}\left(s k_{\mathcal{B}}, i, Q_{i}\right)$ and sends $\left(\sigma_{i}\right)$. $\mathcal{B}$ stores state information $B_{i}=\left(s k_{\mathcal{B}}, T, p k_{\mathcal{B}}, p k_{\mathcal{V}}, a p k, D_{i}^{\prime}\right) . \mathcal{V}$ runs OFVerComp $\left(p k_{\mathcal{B}}, \sigma_{i}, i, Q_{i}\right)$ and, if the output is reject, $\mathcal{V}$ outputs (complete, $i, 0)$ and proceeds with the complaint phase. Otherwise $\mathcal{V}$ stores state information $V_{i}=\left(s k_{\mathcal{V}}, T, p k_{\mathcal{V}}, p k_{\mathcal{B}}, a p k, Q_{i}, \sigma_{i}\right)$, increments the number of the transfer $t=t+1$ and outputs (complete, $i, 1)$.

Vendor complaint. On input the tuple $\left(\operatorname{complain}_{\mathcal{V}}, i\right)$, if no valid request $\left(i, Q_{i}, \omega_{i}\right)$ was previously received, $\mathcal{V}$ does nothing. Otherwise $\mathcal{V}$ runs POTResp $\left(c r s, p k_{\mathcal{V}}, s k_{\mathcal{V}}, Q_{i}\right)$ and sends the request-response pair $\left(\left(i, Q_{i}\right.\right.$, $\left.\left.\omega_{i}\right),(R)\right)$ to $\mathcal{A}$, along with the signature of the previous transfer $(i-1$, $\left.Q_{i-1}, \sigma_{i-1}\right)\left(\left(0, D_{0}, \sigma_{0}\right)\right.$ when $\left.i=1\right)$. $\mathcal{A}$ parses $Q_{i-1}$ to obtain $D_{i-1}$ and runs POTVerReq $\left(c r s, p k_{\mathcal{V}}, D_{i-1}, Q_{i}\right)$, OFVerReq $\left(p k_{\mathcal{B}}, a p k, \omega_{i}, i, Q_{i}\right)$, $\operatorname{OFVerComp}\left(p k_{\mathcal{B}}, \sigma_{i-1}, i-1, Q_{i-1}\right)\left(\operatorname{OFInitV}\left(p k_{\mathcal{B}}, \sigma_{0}, D_{0}\right)\right.$ when $\left.i=1\right)$ and POTVerResp $\left(c r s, p k_{\mathcal{V}}, R\right)$ and, if any of them outputs reject, sets $\sigma_{i}=\perp$ and $R=\perp$ and outputs $\left(\operatorname{complain}_{\mathcal{V}}, i\right.$, inv). Otherwise $\mathcal{A}$ runs $\sigma_{i} \leftarrow \operatorname{OFAdj}\left(p k_{\mathcal{B}}, a s k, a p k, \omega_{i}, i, Q_{i}\right) . \mathcal{A}$ sends $\sigma_{i}$ to $\mathcal{V}$ and $(i, R)$ to $\mathcal{B}$ and outputs (complain $\mathcal{V}, i$, solved). $\mathcal{V}$ runs $\operatorname{OFVerComp}\left(p k_{\mathcal{B}}, \sigma_{i}, i, Q_{i}\right)$ and outputs (compSolved ${ }_{\mathcal{Y}}$ ) if the output is accept. $\mathcal{B}$ runs $\left(m_{\tau}\right) \leftarrow$ POTComplete $\left(\mathrm{crs}, T, R, Q_{i}^{\prime}\right)$, where $Q_{i}^{\prime}$ corresponds to the request for transfer $i$, and outputs $\left(\operatorname{compResp}_{\mathcal{V}}, i, m_{\tau}\right)$.

Buyer complaint. On input $\left(\operatorname{complain}_{\mathcal{B}}, i, \tau\right)$, if a request $\left(i, Q_{i}, \omega_{i}\right)$ was previously computed $\mathcal{B}$ sends it to $\mathcal{A}$. Otherwise, $\mathcal{B}$ sends to $\mathcal{A}$ a new re- 
quest computed by running POTReq and OFReq. $\mathcal{A}$ sends $(i)$ to $\mathcal{V}$, which returns $\left(j, Q_{j}, \sigma_{j}\right)\left(\left(0, D_{0}, \sigma_{0}\right)\right.$ if $\left.i=1\right)$. Then $\mathcal{A}$ proceeds as follows:

- If $\sigma_{j}$ is invalid, $\mathcal{A}$ sends (guilty $\mathcal{V}$ ) to $\mathcal{V}$ and $\mathcal{B}$ and outputs $\left(\right.$ complain $_{\mathcal{B}}, i$, guilty $\left._{\mathcal{V}}\right)$.

- If $\sigma_{j}$ is valid but $j \geq i, \mathcal{A}$ sends $\left(\operatorname{compInv}_{\mathcal{B}}\right)$ to $\mathcal{B}$ and outputs (complain $_{\mathcal{B}}, i$, inv)

- If $\sigma_{j}$ is valid and $j=i-1, \mathcal{A}$ parses $Q_{i-1}$ to obtain $D_{i-1}$, runs POTVerReq $\left(c r s, p k_{\mathcal{V}}, D_{i-1}, Q_{i}\right)$ and, if the output is reject, sends $\left(\operatorname{compInv}_{\mathcal{B}}\right)$ to $\mathcal{B}$ and outputs $\left(\operatorname{complain}_{\mathcal{B}}, i\right.$, inv $) . \mathcal{V}$ (possibly) sends another request $\left(i, Q_{i}^{\prime}, \omega_{i}^{\prime}\right)$. $\mathcal{A}$ verifies it and, if it is correct, but $\left(i, Q_{i}^{\prime}, \omega_{i}^{\prime}\right) \neq\left(i, Q_{i}, \omega_{i}\right), \mathcal{A}$ sends $\left(\operatorname{compInv}_{\mathcal{B}}\right)$ to $\mathcal{B}$ and outputs (complain $_{\mathcal{B}}, i$, inv). Otherwise $\mathcal{A}$ sends $\left(i, Q_{i}, \omega_{i}\right)$ to $\mathcal{V}$. $\mathcal{V}$ verifies the request, runs $(R) \leftarrow \operatorname{POTResp}\left(c r s, T, s k_{\mathcal{V}}, Q_{i}\right)$ and sends $(R)$ to $\mathcal{A}$. $\mathcal{A}$ verifies the response via POTVerResp $\left(c r s, p k_{\mathcal{V}}, R\right)$ and, if it is not valid, $\mathcal{A}$ sends (guilty ${ }_{\mathcal{V}}$ ) to $\mathcal{B}$ and $\mathcal{V}$ and outputs $\left(\operatorname{complain}_{\mathcal{B}}\right.$, $i$, guilty $)_{\mathcal{V}}$. If it is valid, $\mathcal{A}$ sends $R$ to $\mathcal{B}$ and $\sigma_{i} \leftarrow \operatorname{OFAdj}\left(p k_{\mathcal{B}}, a s k\right.$, $\left.a p k, \omega_{i}, i, Q_{i}\right)$ to $\mathcal{V}$, and outputs $\left(\operatorname{complain}_{\mathcal{B}}, i\right.$, solved). $\mathcal{B}$ retrieves $m_{\tau}$ via POTComplete and outputs $\left(\operatorname{compResp}_{\mathcal{B}}, i, m_{\tau}\right)$, while $\mathcal{V}$ outputs compSolved . $_{\mathcal{B}}$

Algorithms POT* correspond to those of the POT schemes (see Section 2.4 and Appendix C). Algorithms $\mathrm{OF}^{*}$ are defined as follows:

OFInitB $\left(\right.$ crs,$\left.D_{0}^{\prime}\right)$ chooses a collision resistant hash function $H:\{0,1\}^{*} \rightarrow \mathbb{Z}_{p}$ and runs $(s k, p k) \leftarrow \operatorname{Kg}\left(1^{\kappa}\right)$ of the VES scheme for the pairing group setup contained in $\mathrm{crs}$. Then it parses $D_{0}^{\prime}$ to obtain the commitment to the deposit $D_{0}$ and signs $\sigma_{0} \leftarrow \operatorname{Sign}\left(s k, H\left(0, D_{0}\right)\right)$. It outputs $s k_{\mathcal{B}}=s k, p k_{\mathcal{B}}=(H, p k)$ and $\sigma_{0}$.

OFInitV $\left(p k_{\mathcal{B}}, \sigma_{0}, D_{0}\right)$ outputs the result of $\mathrm{V} f\left(p k, \sigma_{0}, H\left(0, D_{0}\right)\right)$.

OFInitA $(c r s)$ runs $\operatorname{AdjKg}\left(1^{\kappa}\right)$ and outputs a key pair (ask, apk) for the pairing group setup contained in crs.

$\operatorname{OFReq}\left(s k_{\mathcal{B}}, a p k, i, Q_{i}\right)$ outputs a verifiably encrypted signature $\omega_{i} \leftarrow$ Create $\left(s k_{\mathcal{B}}, a p k, H\left(i, Q_{i}\right)\right)$.

$\operatorname{OFVerReq}\left(p k_{\mathcal{B}}, a p k, \omega_{i}, i, Q_{i}\right)$ outputs the result of $\operatorname{Ves} \bigvee \mathfrak{f}\left(p k, a p k, \omega_{i}, H\left(i, Q_{i}\right)\right)$.

OFComplete $\left(s k_{\mathcal{B}}, i, Q_{i}\right)$ outputs $\sigma_{i} \leftarrow \operatorname{Sign}\left(s k_{\mathcal{B}}, H\left(i, Q_{i}\right)\right)$.

$\operatorname{OFVerComp}\left(p k_{\mathcal{B}}, \sigma_{i}, i, Q_{i}\right)$ outputs $\vee \mathfrak{f}\left(p k, \sigma_{i}, H\left(i, Q_{i}\right)\right)$.

$\operatorname{OFAdj}\left(p k_{\mathcal{B}}, a s k, a p k, \omega_{i}, i, Q_{i}\right)$ outputs $\operatorname{Adj}\left(p k, a s k, a p k, \omega, H\left(i, Q_{i}\right)\right)$.

Theorem 2. This OFPOT scheme securely realizes $\mathcal{F}_{\text {OFPOT }}$.

We prove this theorem in Appendix B. As for efficiency, we note that the overhead in terms of computation and communication cost introduced by the VES scheme are small compared to the cost of the POT scheme. Therefore, a secure POT can efficiently be extended to provide optimistic fair exchange. 


\section{Conclusion}

Our contribution is twofold. First, we have designed a full-simulation secure POT scheme that is more efficient than previous work. Second, we have proposed a generic construction that provides any secure POT scheme with optimistic fair exchange.

We leave open the definition of a more general ideal functionality for fair privacy-preserving e-commerce protocols. Another interesting open problem is the design of a fair POT scheme that is abuse-free in the sense of [39]. Further research also needs to be conducted to show how to integrate e-commerce protocols based on POT with digital rights management systems, and to analyze the compliance of such e-commerce protocols with e-commerce legislation.

\section{Acknowledgements}

This work was supported in part by the IAP Programme P6/26 BCRYPT of the Belgian State. Alfredo Rial is funded by the Research Foundation - Flanders (FWO).

\section{References}

1. Ackerman, M.S., Cranor, L.F., Reagle, J.: Privacy in e-commerce: examining user scenarios and privacy preferences. In: ACM Conference on Electronic Commerce. (1999) 1-8

2. Tsai, J., Egelman, S., Cranor, L., Acquisti, R.: The effect of online privacy information on purchasing behavior: An experimental study, working paper. (June 2007)

3. : Enforcing privacy promises: Section 5 of the ftc act. Federal Trade Commission Act http://www.ftc.gov/privacy/privacyinitiatives/promises.html.

4. Kremer, S.: Formal analysis of optimistic fair exchange protocols (2004)

5. Aiello, W., Ishai, Y., Reingold, O.: Priced oblivious transfer: How to sell digital goods. In Pfitzmann, B., ed.: EUROCRYPT. Volume 2045 of Lecture Notes in Computer Science., Springer (2001) 119-135

6. Tobias, C.: Practical oblivious transfer protocols. In Petitcolas, F.A.P., ed.: Information Hiding. Volume 2578 of Lecture Notes in Computer Science., Springer (2002) 415-426

7. Rial, A., Kohlweiss, M., Preneel, B.: Universally composable adaptive priced oblivious transfer. [40] 231-247

8. Even, S., Goldreich, O., Lempel, A.: A randomized protocol for signing contracts. Commun. ACM 28(6) (1985) 637-647

9. Goldreich, O.: A simple protocol for signing contracts. In: CRYPTO. (1983) 133136

10. Bao, F., Deng, R.H., Mao, W.: Efficient and practical fair exchange protocols with off-line ttp. In: IEEE Symposium on Security and Privacy, IEEE Computer Society (1998) $77-85$

11. Asokan, N., Shoup, V., Waidner, M.: Optimistic fair exchange of digital signatures (extended abstract). In: EUROCRYPT. (1998) 591-606 
12. Avoine, G., Vaudenay, S.: Optimistic fair exchange based on publicly verifiable secret sharing. In Wang, H., Pieprzyk, J., Varadharajan, V., eds.: ACISP. Volume 3108 of Lecture Notes in Computer Science., Springer (2004) 74-85

13. Boneh, D., Gentry, C., Lynn, B., Shacham, H.: Aggregate and verifiably encrypted signatures from bilinear maps. In Biham, E., ed.: EUROCRYPT. Volume 2656 of Lecture Notes in Computer Science., Springer (2003) 416-432

14. Lu, S., Ostrovsky, R., Sahai, A., Shacham, H., Waters, B.: Sequential aggregate signatures and multisignatures without random oracles. In Vaudenay, S., ed.: EUROCRYPT. Volume 4004 of Lecture Notes in Computer Science., Springer (2006) 465-485

15. Rückert, M., Schröder, D.: Security of verifiably encrypted signatures and a construction without random oracles. [40] 17-34

16. Ray, I., Ray, I.: An anomymous fair exchange e-commerce protocol. In: IPDPS, IEEE Computer Society (2001) 172

17. Naor, M., Pinkas, B.: Computationally secure oblivious transfer. J. Cryptology 18(1) (2005) 1-35

18. Camenisch, J., Neven, G., Shelat, A.: Simulatable adaptive oblivious transfer. In Naor, M., ed.: EUROCRYPT. Volume 4515 of Lecture Notes in Computer Science., Springer (2007) 573-590

19. Boneh, D., Boyen, X.: Short signatures without random oracles. [41] 56-73

20. Camenisch, J., Dubovitskaya, M., Neven, G.: Oblivious transfer with access control. In Al-Shaer, E., Jha, S., Keromytis, A.D., eds.: ACM Conference on Computer and Communications Security, ACM (2009) 131-140

21. Boneh, D., Boyen, X., Goh, E.J.: Hierarchical identity based encryption with constant size ciphertext. In Cramer, R., ed.: EUROCRYPT. Volume 3494 of Lecture Notes in Computer Science., Springer (2005) 440-456

22. Goldwasser, S., Micali, S., Rivest, R.L.: A digital signature scheme secure against adaptive chosen-message attacks. SIAM J. Comput. 17(2) (1988) 281-308

23. Pedersen, T.P.: Non-interactive and information-theoretic secure verifiable secret sharing. In: CRYPTO '92. Volume 576 of LNCS. (1992) 129-140

24. Bellare, M., Goldreich, O.: On defining proofs of knowledge. In Brickell, E.F., ed.: CRYPTO '92. Volume 740 of LNCS., Springer-Verlag (1992) 390-420

25. Schnorr, C.P.: Efficient signature generation for smart cards. Journal of Cryptology 4(3) (1991) 239-252

26. Chaum, D., Pedersen, T.P.: Wallet databases with observers. In: CRYPTO '92. Volume 740 of LNCS. (1993) 89-105

27. Camenisch, J., Chaabouni, R., Shelat, A.: Efficient protocols for set membership and range proofs. [51] 234-252

28. Cramer, R., Damgård, I., Schoenmakers, B.: Proofs of partial knowledge and simplified design of witness hiding protocols. In: CRYPTO '94. Volume 839 of LNCS. (1994) 174-187

29. Damgård, I.: Concurrent zero-knowledge is easy in practice. Available online at Theory of Cryptography Library (June 1999)

30. Damgård, I.: On $\sigma$-protocols. Available at http://www.daimi.au.dk/ ivan/ Sigma.ps (2002)

31. Fiat, A., Shamir, A.: How to prove yourself: Practical solutions to identification and signature problems. In Odlyzko, A.M., ed.: CRYPTO '86. Volume 263 of LNCS., Springer Verlag (1987) 186-194

32. Camenisch, J., Stadler, M.: Proof systems for general statements about discrete logarithms. Technical Report TR 260, Institute for Theoretical Computer Science, ETH Zürich (March 1997) 
33. Canetti, R.: Universally composable security: A new paradigm for cryptographic protocols. In: Proc. 42nd IEEE Symposium on Foundations of Computer Science (FOCS). (2001) 136-145

34. Green, M., Hohenberger, S.: Universally composable adaptive oblivious transfer. [51] 179-197

35. Bellare, M., Rogaway, P.: Random oracles are practical: A paradigm for designing efficient protocols. In: First ACM Conference on Computer and Communication Security, Association for Computing Machinery (1993) 62-73

36. Okada, Y., Manabe, Y., Okamoto, T.: An optimistic fair exchange protocol and its security in the universal composability framework. IJACT 1(1) (2008) 70-77

37. Canetti, R.: Obtaining universally compoable security: Towards the bare bones of trust. In: ASIACRYPT. (2007) 88-112

38. Herranz, J.: Restricted adaptive oblivious transfer. Cryptology ePrint Archive, Report 2008/182 (2008) http://eprint.iacr.org/.

39. Garay, J., Jakobsson, M., MacKenzie, P.: Abuse-free optimistic contract signing. In Wiener, M., ed.: CRYPTO '99. Volume 1666 of LNCS., Springer Verlag (1999) 499-466

40. Shacham, H., Waters, B., eds.: Pairing-Based Cryptography - Pairing 2009, Third International Conference, Palo Alto, CA, USA, August 12-14, 2009, Proceedings. In Shacham, H., Waters, B., eds.: Pairing. Volume 5671 of Lecture Notes in Computer Science., Springer (2009)

41. Cachin, C., Camenisch, J., eds.: Advances in Cryptology - EUROCRYPT 2004, International Conference on the Theory and Applications of Cryptographic Techniques, Interlaken, Switzerland, May 2-6, 2004, Proceedings. In Cachin, C., Camenisch, J., eds.: EUROCRYPT. Volume 3027 of Lecture Notes in Computer Science., Springer (2004)

42. Boyen, X., Waters, B.: Full-domain subgroup hiding and constant-size group signatures. In Okamoto, T., Wang, X., eds.: Public Key Cryptography. Volume 4450 of Lecture Notes in Computer Science., Springer (2007) 1-15

43. Belenkiy, M., Chase, M., Kohlweiss, M., Lysyanskaya, A.: P-signatures and noninteractive anonymous credentials. In Canetti, R., ed.: TCC. Volume 4948 of Lecture Notes in Computer Science., Springer (2008) 356-374

44. Boneh, D., Boyen, X., Shacham, H.: Short group signatures. In Franklin, M.K., ed.: CRYPTO. Volume 3152 of Lecture Notes in Computer Science., Springer (2004) $41-55$

45. Santis, A.D., Di Crescenzo, G., Persiano, G.: Necessary and sufficient assumptions for non-interactive zero-knowledge proofs of knowledge for all NP relations. In Montanari, U., Rolim, J.P., Welzl, E., eds.: Proc. 27th International Colloquium on Automata, Languages and Programming (ICALP). Volume 1853 of LNCS., Springer Verlag (2000) 451-462

46. Goldwasser, S., Micali, S., Rackoff, C.: The knowledge complexity of interactive proof systems. SIAM J. Comput. 18(1) (1989) 186-208

47. Goldreich, O.: Foundations of Cryptography: Basic Tools. Cambridge University Press, New York, NY, USA (2000)

48. Blum, M., Feldman, P., Micali, S.: Non-interactive zero-knowledge and its applications. In: STOC '88: Proceedings of the twentieth annual ACM symposium on Theory of computing, New York, NY, USA, ACM Press (1988) 103-112

49. Feige, U., Lapidot, D., Shamir, A.: Multiple noninteractive zero knowledge proofs under general assumptions. SIAM Journal on Computing 29(1) (1999) 1-28 
50. Groth, J., Sahai, A.: Efficient non-interactive proof systems for bilinear groups. In Smart, N.P., ed.: EUROCRYPT. Volume 4965 of Lecture Notes in Computer Science., Springer (2008) 415-432

51. Pieprzyk, J., ed.: Advances in Cryptology - ASIACRYPT 2008, 14th International Conference on the Theory and Application of Cryptology and Information Security, Melbourne, Australia, December 7-11, 2008. Proceedings. In Pieprzyk, J., ed.: ASIACRYPT. Volume 5350 of Lecture Notes in Computer Science., Springer (2008)

\section{A Security Proof of POT scheme}

In order to prove Theorem 1 , we need to build a simulator $\mathcal{S}^{\prime}=\left\{\mathcal{S}_{\mathcal{V}}^{\prime}, \mathcal{S}_{\mathcal{B}}^{\prime}\right\}$ that invokes a copy of adversary $\mathcal{E}$ and interacts with $\mathcal{F}_{\text {POT }}$ and environment $\mathcal{Z}$ in such a way that ensembles IDEAL $\operatorname{F}_{\mathcal{F}_{\mathrm{POT}}, \mathcal{S}^{\prime}, \mathcal{Z}}$ and $\mathrm{REAL}_{\mathrm{POT}, \mathcal{E}, \mathcal{Z}}$ are computationally indistinguishable.

Simulation of buyer's security. In this case only the vendor $\mathcal{V}$ is corrupted.

1. Upon receiving message $\left(p k_{\mathcal{V}}, T\right)$ from $\mathcal{E}, \mathcal{S}_{\mathcal{V}}^{\prime}$ verifies $p k_{\mathcal{V}}$ and $T$ as described in POTInitB. Then $\mathcal{S}_{\mathcal{V}}^{\prime}$ parses $p k_{\mathcal{V}}$ as $\left(\Phi, H\right.$, par $\left._{\text {ran }}, p k, p k_{\text {enc }}, \pi_{1}\right)$ and extracts the witness $h$ from $\pi_{1}$ (via forking lemma). $\mathcal{S}_{\mathcal{V}}^{\prime}$ parses $T$ as $\left(C_{1}, \ldots, C_{N}\right)$ and, for $i=1$ to $N$, parses $C_{i}$ as $\left(A_{i}, B_{i}, p_{i}\right)$ and computes $m_{i}=B_{i} / e\left(h, A_{i}\right)$. $\mathcal{S}_{\mathcal{V}}^{\prime}$ sends (init, $\left.m_{1}, p_{1}, \ldots, m_{N}, p_{N}\right)$ to $\mathcal{F}_{\mathrm{POT}}$.

2. Upon receiving (deposit, $\left.a c_{0}\right)$ from $\mathcal{F}_{\mathrm{POT}}, \mathcal{S}_{\mathcal{V}}^{\prime}$ picks open ac $_{0}$, sets $P=($ Enc $\left(p k_{e n c}, a c_{0}\right)$,open oc $\left._{0}\right)$, stores $D_{0}^{\prime}=\left(a c_{0}\right.$, open $_{a c_{0}}, D_{0}=\operatorname{PECom}\left(\right.$ par $_{c o m}, a c_{0}$, open $\left._{a c_{0}}\right)$ ) and sends $(P)$ to $\mathcal{E}$.

3. Upon receiving (request) from $\mathcal{F}_{\mathrm{POT}}, \mathcal{S}_{\mathcal{V}}^{\prime}$ selects $\tau_{\text {min }}$ that corresponds to the item with the lowest price, and runs POTReq $\left(p k_{\mathcal{V}}, T, D_{i-1}^{\prime}, \tau_{\text {min }}\right)$ to obtain $Q, Q^{\prime}$ and $D_{i}^{\prime} . \mathcal{S}_{\mathcal{V}}^{\prime}$ sends $(Q)$ to $\mathcal{E}$.

4. Upon receiving $(R)$ from $\mathcal{E}, \mathcal{S}_{\mathcal{V}}^{\prime}$ verifies $\pi_{3}$. If the proof is correct, $\mathcal{S}_{\mathcal{V}}^{\prime}$ sends (response, 1) to $\mathcal{F}_{\mathrm{POT}}$. Otherwise $\mathcal{S}_{\mathcal{V}}^{\prime}$ sends (response, 0) to $\mathcal{F}_{\mathrm{POT}}$.

Claim. When only $\mathcal{V}$ is corrupted, the distribution ensembles $\operatorname{IDEAL}_{\mathcal{F}_{\mathrm{POT}}, \mathcal{S}_{\mathcal{V}}^{\prime}, \mathcal{Z}}$ and $\operatorname{REAL}_{\mathrm{POT}, \mathcal{E}, \mathcal{Z}}$ are indistinguishable under the hiding property of the commitment scheme.

Proof. We show by means of a series of hybrid games that the environment $\mathcal{Z}$ cannot distinguish between the real execution ensemble $\operatorname{REAL}_{\mathrm{POT}, \mathcal{E}, \mathcal{Z}}$ and the simulated ensemble IDEAL $\mathcal{F}_{\mathcal{F}_{\mathrm{POT}}, \mathcal{S}_{\nu}^{\prime}, \mathcal{Z}}$ with non-negligible probability. We denote by $\operatorname{Pr}[$ Game $i]$ the probability that $\mathcal{Z}$ distinguishes between the ensemble of Game $i$ and that of the real execution.

Game 0: This game corresponds to the execution of the real-world protocol with an honest $\mathcal{B}$. Therefore, $\operatorname{Pr}[$ Game 0$]=0$.

Game 1: This game proceeds as Game 0, except that we use the knowledge extractor to obtain $h$ from $\pi_{1}$. If extraction fails, we abort. The probability that extraction fails is negligible, and thus $\mid \operatorname{Pr}[$ Game 1] $-\operatorname{Pr}[$ Game 0] $\mid=$ $\nu_{1}$. 
Game 2: This game proceeds as Game 1, except that $P=\operatorname{Enc}\left(p k_{\text {enc }}, a c_{0}\right)$ is replaced by another valid encryption of the same value under the same public key. Therefore $\mid \operatorname{Pr}[$ Game 2] $-\operatorname{Pr}[$ Game 1] $\mid=0$.

Game 3: This game proceeds as Game 2, except that buyer's requests are replaced by other valid requests that use a fixed $\tau_{m i n}$, which corresponds to the lowest price. Therefore $Q=\left(V, D_{p}, D_{i}, \pi_{2}\right)$ is computed as follows: $V=$ $A_{\tau_{\text {min }}}^{v}$ for a random $v ; D_{p}=\operatorname{PECom}\left(\right.$ par $_{\text {com }}, p_{\tau}$, open $\left._{p}\right)$; and $D_{i}=$ PECom $\left(\right.$ par $_{\text {com }}, a c_{i}$, open $\left._{a c_{i}}\right)$ for $a c_{i}=a c_{i-1}-p_{\tau}$. We note that $V$ completely hides $A_{\tau_{m i n}}$ and that $D_{p}$ and $D_{i}$ completely hide $p$ and $a c_{i}$ because Pedersen commitments are information theoretically hiding. Moreover, the proof of knowledge $\pi_{2}$ is zero-knowledge and therefore does not leak any information about the witness. Therefore, $\mid \operatorname{Pr}[$ Game 3] $-\operatorname{Pr}[$ Game 2] $\mid=0$.

$\mathcal{S}_{\mathcal{V}}^{\prime}$ performs all the changes described in Game 3, and forwards and receives messages from $\mathcal{F}_{P O T}$ as described in our simulation. The distribution produced in Game 3 is identical to that of our simulation. Therefore, by summation we have that $\mid \operatorname{Pr}\left[\right.$ Game 3] $\leq \nu_{1}$.

Simulation of vendor's security. In this case only the buyer $\mathcal{B}$ is corrupted.

1. Upon receiving (init, $p_{1}, \ldots, p_{N}$ ) from $\mathcal{F}_{\mathrm{POT}}$, for $i=1$ to $N, \mathcal{S}_{\mathcal{B}}^{\prime}$ chooses random messages $m_{i}^{\prime} \leftarrow \mathbb{G}_{t}$ and follows algorithm POTInitV $\left(1^{\kappa}, m_{1}^{\prime}, p_{1}, \ldots, m_{N}^{\prime}\right.$, $\left.p_{N}, D_{\max }\right)$ to compute a database commitment $T, p k_{\mathcal{V}}$ and $s k_{\mathcal{V}} . \mathcal{S}_{\mathcal{B}}^{\prime}$ sends $\left(p k_{\mathcal{V}}, T\right)$ to $\mathcal{E}$.

2. Upon receiving $(P)$ from $\mathcal{E}, \mathcal{S}_{\mathcal{B}}^{\prime}$ follows algorithm POTGetDep $\left(s k_{\mathcal{V}}, P, D_{\text {max }}\right)$ to obtain $\left(D_{0}, a c_{0}\right)$ and sends (deposit, $\left.a c_{0}\right)$ to $\mathcal{F}_{\mathrm{POT}}$.

3. Upon receiving $(Q), \mathcal{S}_{\mathcal{B}}^{\prime}$ parses $Q$ as $\left(V, D_{p}, D_{i}, \pi_{2}\right)$. $\mathcal{S}_{\mathcal{B}}^{\prime}$ verifies $\pi_{2}$ and extracts the witness $\left(p_{\tau}\right.$, open $_{p}, a c_{i}$, open $_{a c_{i}}, a c_{i-1}$, open $\left._{a c_{i-1}}, v, \alpha\right)$. If $p_{\tau}$ is not a valid price, $\mathcal{S}_{\mathcal{B}}^{\prime}$ aborts. Otherwise $\mathcal{S}_{\mathcal{B}}^{\prime}$ maps $p_{\tau}$ to $\tau$. Then $\mathcal{S}_{\mathcal{B}}^{\prime}$ checks that the extracted $a c_{i}$ is correct, i.e., that it equals $a c_{i-1}-p_{\tau}$, and aborts if it is not the case. $\mathcal{S}_{\mathcal{B}}^{\prime}$ sends (request, $\tau$ ) to $\mathcal{F}_{\mathrm{POT}}$.

4. Upon receiving (response, $\left.m_{\tau}\right)$ from $\mathcal{F}_{\mathrm{POT}}, \mathcal{S}_{\mathcal{B}}^{\prime}$ parses $T$ as $\left(C_{1}, \ldots, C_{N}\right)$ and $C_{\tau}$ as $\left(A_{\tau}, B_{\tau}, p_{\tau}\right) . \mathcal{S}_{\mathcal{B}}^{\prime}$ sets $W=\left(B_{\sigma} / m_{\tau}\right)^{v}$ and computes a simulated proof $\pi_{3} . \mathcal{S}_{\mathcal{B}}^{\prime}$ sends $R=\left(W, \pi_{3}\right)$ to $\mathcal{E}$.

Theorem 3. When only the buyer $\mathcal{B}$ is corrupted, the distribution ensembles $\mathrm{IDEAL}_{\mathcal{F}_{\mathrm{POT}}, \mathcal{S}_{\mathcal{B}}^{\prime}, \mathcal{Z}}$ and $\mathrm{REAL} \mathrm{POT}, \mathcal{E}, \mathcal{Z}$ are computationally indistinguishable under the $(N+1, d+1)-S D H$ assumption and the $(N+1)-P D D H$ assumption, and under the binding property of the commitment scheme.

Proof.

Game 0: This game corresponds to the execution of the real-world protocol with an honest $\mathcal{V}$. Therefore, $\operatorname{Pr}[$ Game 0$]=0$.

Game 1: This game is identical to Game 0, except that we use the knowledge extractor of $\pi_{2}$ to obtain witness $\left(p_{\tau}\right.$, open $_{p}, a c_{i}$, open $_{a c_{i}}, a c_{i-1}$, open $_{a c_{i-1}}, v$, $\alpha$ ). If extraction fails, we abort. The probability that extraction fails is negligible. Therefore $\mid \operatorname{Pr}\left[\right.$ Game 1] $-\operatorname{Pr}\left[\right.$ Game 0] $\mid=\nu_{1}$. 
Game 2: This game is identical to Game 1, except that we abort when the extracted $p_{\tau}$ is not a valid price. The probability that $\mathcal{Z}$ distinguishes between Game 2 and Game 1 is bounded by the following theorem:

Theorem 4. If the $(N+1)-S D H$ assumption holds, then $\mid \operatorname{Pr}[$ Game 2]$\operatorname{Pr}\left[\right.$ Game 1] $\mid=\nu_{2}$.

Proof. We build an algorithm $\mathcal{T}$ that, given and adversary $\mathcal{E}$ that outputs a valid proof $\pi_{2}$ for which an invalid price is extracted with non-negligible probability, breaks the weak unforgeability property of the signature scheme in [19] with non-negligible probability. Given $\mathcal{T}$, it is shown in [19] how to break the SDH assumption.

Upon receiving (init, $\left.p_{1}, \ldots, p_{N}\right)$ from $\mathcal{F}_{\mathrm{POT}}, \mathcal{T}$ sends $\left(p_{1}, \ldots, p_{N}\right)$ to the challenger and receives the public key $p k$ and signatures on messages $\left(p_{1}, \ldots\right.$, $\left.p_{N}\right)$ from the challenger. $\mathcal{T}$ employs these signatures to compute $T$. Upon receiving a request $Q=\left(V, D_{p}, D_{i}, \pi_{2}\right), \mathcal{T}$ extracts from $\pi_{2}$ the witness $\left(p_{\tau}\right.$, open $_{p}, a c_{i}$, open $_{a c_{i}}, a c_{i-1}$, open $\left._{a c_{i-1}}, v, \alpha\right)$. If $p_{\tau}$ is not in the list $\left(p_{1}, \ldots, p_{N}\right)$, $\mathcal{T}$ outputs $\left(p_{\tau}, V^{1 / v}\right)$ as its forgery.

Game 3: This game is identical to Game 2, except that we abort when the extracted value $a c_{i}$ does not equal $a c_{i-1}-p_{\tau}$. This means that $\mathcal{E}$ was able to break the binding property of Pedersen commitments, which holds under the discrete logarithm assumption. Therefore, the probability that $\mathcal{Z}$ distinguishes between Game 3 and Game 2 is bounded by the following theorem:

Theorem 5. If the commitment scheme is binding, then $\mid \operatorname{Pr}[$ Game 3] $\operatorname{Pr}\left[\right.$ Game 2] $=\nu_{3}$.

Proof. We build an algorithm $\mathcal{T}$ that breaks the binding property of the commitment scheme with non-negligible probability. Given $\mathcal{T}$, it is shown in [23] how to break the discrete logarithm assumption.

Upon receiving the parameters par $_{\text {com }}$ of the commitment scheme from the challenger, $\mathcal{T}$ uses them to compute the database commitment $T$. Upon receiving a request $Q=\left(V, D_{p}, D_{i}, \pi_{2}\right), \mathcal{T}$ extracts $\left(p_{\tau}\right.$, open $_{p}$, ac $_{i}^{\prime}$, open ac $_{i}^{\prime}$, $v, \alpha)$ from $\pi_{2}$. $\mathcal{T}$ knows $\left(a c_{i-1}\right.$, open $\left._{a c_{i-1}}\right)$ from extraction of the previous request. If $a c_{i}^{\prime}$ does not equal $a c_{i-1}-p_{\sigma}, \mathcal{T}$, given commitment $D_{i}=$ $\tilde{g}^{a c_{i}^{\prime}} \tilde{h}^{\text {open }_{a c_{i}^{\prime}}}$, is able to open $D_{i}$ to $\left(a c_{i}\right.$ open $\left._{a c_{i}}\right)$, where $a c_{i}=a c_{i-1}-p_{\tau}$ and open $_{a c_{i}}=$ open $_{a c_{i-1}}-$ open $_{p}-\alpha$ (we recall that $\alpha$ fulfills this equality

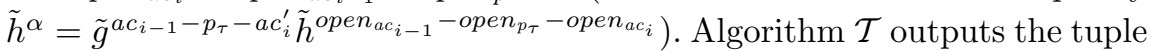
$\left(D_{i}, a c_{i}\right.$, open $_{a c_{i}}, a c_{i}^{\prime}$, open $\left._{a c_{i}}^{\prime}\right)$ to break the binding property of the commitment scheme.

Game 4: This game is identical to Game 3, except that we abort if $a c_{i} \notin$ $\left[0, D_{\max }=d^{a}\right)$. The probability that $\mathcal{Z}$ distinguishes between Game 4 and Game 3 is bounded by the following theorem:

Theorem 6. If the $(d+1)-S D H$ assumption holds, then $\mid \operatorname{Pr}[$ Game 4] $\operatorname{Pr}\left[\right.$ Game 3]| $=\nu_{4}$. 
Proof. We build an algorithm $\mathcal{T}$ that breaks the weak unforgeability property of the signature scheme in [19] with non-negligible probability. Given $\mathcal{T}$, it is shown in [19] how to break the SDH assumption.

Given $D_{\max }=d^{a}$, when running $\operatorname{Init} \operatorname{Ver}\left(1^{\kappa}, D_{\max }\right) \mathcal{T}$ sends values $i \in \mathbb{Z}_{d}$ to the challenger and receives from the challenger the public key $p k$ and signatures on values $i \in \mathbb{Z}_{d}$, and uses them to compute $p a r_{\text {ran }}$. Upon receiving a request, $\mathcal{T}$ gets the values $\left(\left\{V_{j}\right\}_{j \in \mathbb{Z}_{a}}\right.$, pok $)$ of the range proof and extracts the witness $\left(\right.$ open $_{\alpha},\left\{\alpha_{j}, v_{j}\right\}_{j \in \mathbb{Z}_{a}}$ ). (We recall that the range proof consists of $\left(\left\{V_{j}\right\}_{j \in \mathbb{Z}_{a}}\right.$, pok $)$, where proof pok $=\operatorname{NIPK}\left\{\left(\right.\right.$ open $\left._{\alpha},\left\{\alpha_{j}, v_{j}\right\}_{j \in \mathbb{Z}_{a}}\right)$ : $\left.C=h^{o p e n} \prod_{j \in \mathbb{Z}_{a}}\left(g^{u^{j}}\right)^{\alpha_{j}} \wedge V_{j}=g^{v_{j} /\left(s k+\alpha_{j}\right)}\right\}$.) If $a c_{i}=\sum_{j \in \mathbb{Z}_{a}} \alpha_{j} d^{j}$ is not in $\left[0, D_{\text {max }}\right)$, there must be a value $\alpha_{j} \notin \mathbb{Z}_{d} . \mathcal{T}$ outputs $\left(\alpha_{j}, V_{j}^{1 / v_{j}}\right)$ as its forgery.

Game 5: This game is identical to Game 4, except that now the database commitment $T^{\prime}$ is computed by using random messages. At this point $\pi_{3}$ is replaced by a simulated proof of a false statement. The probability that $\mathcal{Z}$ distinguishes between Game 5 and Game 4 is bounded by the following theorem:

Theorem 7. If the $(N+1)-P D D H$ assumption holds, $\mid \operatorname{Pr}[$ Game 5]$\operatorname{Pr}[$ Game 4$] \mid=\nu_{5}$.

Proof. Given an adversary $\mathcal{E}$ which distinguishes between Game 5 and Game 4 with non-negligible probability, we construct an algorithm $\mathcal{T}$ that breaks the $(N+1)$-PDDH assumption as follows. $\mathcal{T}$ receives the vector $\left(u, u^{x}, \ldots, u^{x^{N+1}}, V\right) \in \mathbb{G}^{N+2} \times \mathbb{G}_{t}$, where $x \in \mathbb{Z}_{p}$, and the vector $\left(T_{1}, \ldots\right.$, $\left.T_{N+1}\right) \in \mathbb{G}_{t}^{N+1}$. Let $T_{0}=V$ and $f$ be the polynomial defined as $f(X)=$ $\prod_{i=1}^{N}\left(X+p_{i}\right)=\sum_{i=0}^{N} c_{i} X^{i}$. Then $\mathcal{T}$ sets $g \leftarrow u^{f(x)}=\prod_{i=0}^{N}\left(u^{x^{i}}\right)^{c_{i}}$ and $y \leftarrow$ $g^{x}=\prod_{i=0}^{N}\left(u^{x^{i+1}}\right)^{c_{i}}$. If $f_{i}$ is the polynomial defined by $f_{i}(X)=f(X) /(X+$ $\left.p_{i}\right)=\sum_{j=0}^{N-1} c_{i, j} X^{j}$, then $\mathcal{T}$ can also compute the values $A_{i}=g^{1 /\left(x+p_{i}\right)}$ as $A_{i} \leftarrow \prod_{j=0}^{N-1}\left(u^{x^{j}}\right)^{c_{i, j}} . \mathcal{T}$ then sets $H \leftarrow V^{f(x)}=\prod_{i=0}^{N} T_{i}^{c_{i}}$, and computes $B_{i}$ as $H^{1 /\left(x+p_{i}\right)}$ as $B_{i} \leftarrow \prod_{j=0}^{N-1} T_{i}^{c_{i, j}}$, and continues the simulation. One can check that if $T_{i}=V^{x^{i}}$ then the simulation corresponds to Game 4, while if $\left(T_{1}, \ldots, T_{N+1}\right)$ is random then the simulation corresponds to Game 5 . Therefore, if $\mathcal{E}$ can distinguish between both, then $\mathcal{T}$ can break the $(N+1)$ PDDH assumption.

$\mathcal{S}_{\mathcal{B}}^{\prime}$ performs all the changes described in Game 5, and forwards and receives messages from $\mathcal{F}_{P O T}$ as described in our simulation. The distribution produced in Game 5 is identical to that of our simulation. Therefore, by summation we have that $\mid \operatorname{Pr}\left[\right.$ Game 5] $\leq \nu_{6}$.

\section{B Security Proofs for OFPOT Scheme}

In order to prove Theorem 2, we need to build a simulator $\mathcal{S}=\left\{\mathcal{S}_{\mathcal{V}}, \mathcal{S}_{\mathcal{B}}\right\}$ that invokes a copy of adversary $\mathcal{E}$ and interacts with $\mathcal{F}_{\text {OFPOT }}$ and environment $\mathcal{Z}$ in 
such a way that ensembles $\operatorname{IDEAL}_{\mathcal{F}_{\mathrm{OFPOT}}, \mathcal{S}, \mathcal{Z}}$ and $\mathrm{REAL}_{\mathrm{OFPOT}, \mathcal{E}, \mathcal{Z}}$ are computationally indistinguishable. Our simulator will use the simulators $\mathcal{S}^{\prime}=\left\{\mathcal{S}_{\mathcal{V}}^{\prime}, \mathcal{S}_{\mathcal{B}}^{\prime}\right\}$ of the POT schemes, which are described in Appendix A and in Appendix C.3.

Simulation of buyer's security. In this case only the vendor $\mathcal{V}$ is corrupted.

- Upon receiving $(\mathrm{crs})$ from $\mathcal{E}, \mathcal{S}_{\mathcal{V}}$ runs $\mathcal{S}_{\mathcal{V}}^{\prime}$ on input (crs) to get (crs) and sends (crs, crs) to $\mathcal{E}$.

- Upon receiving $\left(p k_{\mathcal{V}}, T\right)$ from $\mathcal{E}, \mathcal{S}_{\mathcal{V}}$ runs $\mathcal{S}_{\mathcal{V}}^{\prime}$ on input $\left(p k_{\mathcal{V}}, T\right)$ to get (init, $\left.m_{1}, p_{1}, \ldots, m_{N}, p_{N}\right)$ and sends (init, $\left.m_{1}, p_{1}, \ldots, m_{N}, p_{N}\right)$ to $\mathcal{F}_{\text {OFPOт }}$.

- Upon receiving (deposit, $a c_{0}$ ) from $\mathcal{F}_{\text {OFPOT }}, \mathcal{S}_{\mathcal{V}}$ runs $\mathcal{S}_{\mathcal{V}}^{\prime}$ on input (deposit, $\left.a c_{0}\right)$ to get $\left(P, D_{0}^{\prime}\right) . \mathcal{S}_{\mathcal{V}}$ runs $\left(s k_{\mathcal{B}}, p k_{\mathcal{B}}, \sigma_{0}\right) \leftarrow$ OFInitB $\left(\right.$ crs,$\left.D_{0}^{\prime}\right)$ and sends $\left(P, p k_{\mathcal{B}}, \sigma_{0}\right)$ to $\mathcal{E}$.

- Upon receiving a request from $\mathcal{E}, \mathcal{S}_{\mathcal{V}}$ executes $(a s k, a p k) \leftarrow$ OFInitA $(c r s)$ and sends apk to $\mathcal{E}$.

- Upon receiving $\left(p k_{\mathcal{V}}, p k_{\mathcal{B}}\right)$ from $\mathcal{E}, \mathcal{S}_{\mathcal{V}}$ aborts if they do not equal $p k_{\mathcal{V}}$ previously sent by $\mathcal{E}$ or $p k_{\mathcal{B}}$ previously sent to $\mathcal{E}{ }^{8}$

- Upon receiving (request, $i$ ) from $\mathcal{F}_{\text {OFPOT }}, \mathcal{S}_{\mathcal{V}}$ runs $\mathcal{S}_{\mathcal{V}}^{\prime}$ on input (request) to obtain $\left(Q_{i}, D_{i}^{\prime}\right) . \mathcal{S}_{\mathcal{V}}$ runs $\omega_{i} \leftarrow \operatorname{OFReq}\left(s k_{\mathcal{B}}, a p k, i, Q_{i}\right)$ and sends $\left(i, Q_{i}, \omega_{i}\right)$ to $\mathcal{E}$.

- Upon receiving $R$ from $\mathcal{E}, \mathcal{S}_{\mathcal{V}}$ runs $\mathcal{S}_{\mathcal{V}}^{\prime}$ on input $R$ to obtain a bit $b$, and sends (response, $i, b)$ to $\mathcal{F}_{\text {OFPOT }}$.

- Upon receiving (complete, $i, c)$ from $\mathcal{F}_{\text {OFPOT }}$, if $c=0 \mathcal{S}_{\mathcal{V}}$ sets $\sigma_{i}=\perp$. Otherwise $\mathcal{S}_{\mathcal{V}}$ runs $\sigma_{i} \leftarrow$ OFComplete $\left(s k_{\mathcal{B}}, i, Q_{i}\right) . \mathcal{S}_{\mathcal{V}}$ sends $\sigma_{i}$ to $\mathcal{E}$.

- Upon receiving a complain $\left(\left(i, Q_{i}, \omega_{i}\right),(R),\left(i-1, Q_{i-1}, \sigma_{i-1}\right)\right)\left(\left(0, D_{0}, \sigma_{0}\right)\right.$ when $i=1)$ from $\mathcal{E}, \mathcal{S}_{\mathcal{V}}$ parses $Q_{i-1}$ to get $D_{i-1}$ and runs POTVerReq $(\mathrm{crs}$, $\left.p k_{\mathcal{V}}, D_{i-1}, Q_{i}\right)$, OFVerReq $\left(p k_{\mathcal{B}}, a p k, \omega_{i}, i, Q_{i}\right), \operatorname{OFVerComp}\left(p k_{\mathcal{B}}, \sigma_{i-1}, i-1\right.$, $\left.Q_{i-1}\right)$ (OFInitV $\left(p k_{\mathcal{B}}, \sigma_{0}, D_{0}\right)$ when $i=1$ ) and POTVerResp $\left(c r s, p k_{\mathcal{V}}, R\right)$, and ignores the complaint if any of the algorithms outputs reject. If all the algorithms output accept and $\mathcal{E}$ did not receive before a signature on $H(i-$ $\left.1, Q_{i-1}\right)$ or a verifiably encrypted signature on $H\left(i, Q_{i}\right), \mathcal{S}_{\mathcal{V}}$ aborts. Otherwise, $\mathcal{S}_{\mathcal{V}}$ sends $\left(\operatorname{complain}_{\mathcal{V}}, i\right)$ to $\mathcal{F}_{\text {OFPOT }}$.

- Upon receiving $\left(\right.$ compInv $\left._{\mathcal{V}}\right)$ from $\mathcal{F}_{\text {OFPOT }}, \mathcal{S}_{\mathcal{V}}$ forwards $\left(\operatorname{compInv}_{\mathcal{V}}\right)$ to $\mathcal{E}$.

- Upon receiving (compSolved ${ }_{\mathcal{V}}$ ) from $\mathcal{F}_{\text {OFPOT }}, \mathcal{S}_{\mathcal{V}}$ executes $\sigma_{i} \leftarrow$ OFComplete $\left(s k_{\mathcal{B}}, i, Q_{i}\right)$ and sends $\sigma_{i}$ to $\mathcal{E}$.

- Upon receiving $\left(\operatorname{complain}_{\mathcal{B}}, i\right)$ from $\mathcal{F}_{\text {OFPOT }}, \mathcal{S}_{\mathcal{V}}$ sends $\left(\operatorname{complain}_{\mathcal{B}}, i\right)$ to $\mathcal{E}$.

- Upon receiving a signature $\left(j, Q_{j}, \sigma_{j}\right)$ from $\mathcal{E}, \mathcal{S}_{\mathcal{V}}$ proceeds as follows:

- If the signature is invalid, $\mathcal{S}_{\mathcal{V}}$ sends (collaborate, $\left.i, 0\right)$ to $\mathcal{F}_{\text {OFPOT. }}$

- If the signature is valid and $j \geq i, \mathcal{S}_{\mathcal{V}}$ aborts.

- If it is valid and $j=i-1, \mathcal{S}_{\mathcal{V}}$ checks the request $\left(i, Q_{i}^{\prime}, \omega_{i}^{\prime}\right)$ (possibly) sent by $\mathcal{E}$ and aborts if it is correct but $\mathcal{E}$ was not given a verifiably encrypted signature on $H\left(i, Q_{i}^{\prime}\right)$. Otherwise $\mathcal{S}_{\mathcal{V}}$ runs $\mathcal{S}_{\mathcal{V}}^{\prime}$ on input (request) to obtain $\left(Q_{i}, D_{i}^{\prime}\right) . \mathcal{S}_{\mathcal{V}}$ runs $\omega_{i} \leftarrow \operatorname{OFReq}\left(s k_{\mathcal{B}}, a p k, i, Q_{i}\right)$ and sends $\left(i, Q_{i}, \omega_{i}\right)$ to $\mathcal{E}$. Upon receiving $R$ from $\mathcal{E}, \mathcal{S}_{\mathcal{V}}$ verifies the response. If it is not correct, $\mathcal{S}_{\mathcal{V}}$ sends (collaborate, $\left.i, 0\right)$ to $\mathcal{F}_{\text {OFPOT }}$, and otherwise (collaborate, $i, 1$ ).

\footnotetext{
${ }^{8}$ We recall that adjudicator $\mathcal{A}$ checks equality between the public keys sent by $\mathcal{V}$ and $\mathcal{B}$, and aborts if they are not equal.
} 


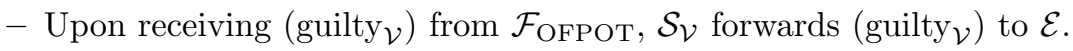

- Upon receiving $\left(\right.$ compSolved $_{\mathcal{B}}$ ) from $\mathcal{F}_{\text {OFPOT }}, \mathcal{S}_{\mathcal{V}}$ executes $\sigma_{i} \leftarrow$ OFComplete $\left(s k_{\mathcal{B}}, i, Q_{i}\right) . \mathcal{S}_{\mathcal{V}}$ sends $\sigma_{i}$ to $\mathcal{E}$.

Claim. When only $\mathcal{V}$ is corrupted, the distribution ensembles $\operatorname{IDEAL}_{\mathcal{F}_{\text {Ӧрот }}, \mathcal{S}, \mathcal{Z}}$ and $\mathrm{REAL}_{\mathrm{OFPOT}, \mathcal{E}, \mathcal{Z}}$ are computationally indistinguishable under the assumption that buyer's security in the POT scheme holds, under the unforgeability and opacity properties of the VES scheme, and under the assumption that $H$ is collision-resistant.

We show by means of a series of hybrid games that the environment $\mathcal{Z}$ cannot distinguish between the real execution ensemble $\mathrm{REAL}_{\mathrm{OFPOT}, \mathcal{E}, \mathcal{Z}}$ and the simulated ensemble IDEAL $\operatorname{F}_{\mathcal{F}_{\text {ОFР }}, \mathcal{S}, \mathcal{Z}}$ with non-negligible probability. We denote by $\operatorname{Pr}[$ Game $i]$ the probability that $\mathcal{Z}$ distinguishes between the ensemble of Game $i$ and that of the real execution.

Game 0: This game corresponds to the execution of the real-world protocol with honest $\mathcal{B}$ and $\mathcal{A}$. Therefore, $\operatorname{Pr}[$ Game 0$]=0$.

Game 1: This game performs all the changes described in $\mathcal{S}_{\mathcal{V}}^{\prime}$. The security proof of the POT scheme demonstrates that $\mid \operatorname{Pr}[$ Game 1$]-\operatorname{Pr}[$ Game 0$] \mid=$ $\nu_{1}$.

Game 2: This game proceeds as Game 1, except that the public keys $p k_{\mathcal{B}}$ and apk are replaced by other valid public keys $p k_{\mathcal{B}}^{\prime}$ and $a p k^{\prime}$, and signatures $\omega$ and $\sigma$ are computed on input $p k_{\mathcal{B}}^{\prime}$ and $a p k^{\prime}$. Since $p k_{\mathcal{B}}^{\prime}$ and $a p k^{\prime}$ follow the same distribution as $p k_{\mathcal{B}}$ and $a p k, \mid \operatorname{Pr}[$ Game 2] $-\operatorname{Pr}[$ Game 1] $\mid=0$.

Game 3: This game proceeds as Game 2, except that we abort if $\mathcal{E}$ sends a verifiably encrypted signature $\omega_{i}$ on a pair $\left(i, Q_{i}\right)$ and $\mathcal{E}$ did not receive before a verifiably encrypted signature on $\left(i, Q_{i}\right)$. The probability that environment $\mathcal{Z}$ distinguishes between Game 3 and Game 2 is bounded by the following theorem:

Theorem 8. If the VES scheme is unforgeable and $H$ is a collision resistant hash function, $\mid \operatorname{Pr}\left[\right.$ Game 3] $-\operatorname{Pr}\left[\right.$ Game 2] $\mid=\nu_{2}$.

Proof. Given an adversary $\mathcal{E}$ that outputs $\omega_{i}$ on a new pair $\left(i, Q_{i}\right)$ with non-negligible probability, we construct an algorithm $\mathcal{T}$ that either breaks the collision resistance property of $H$ or wins the unforgeability game of the VES scheme (see [15]) with non-negligible probability. Upon receiving apk and $p k$ from the challenger $\mathcal{C}_{u}$ of the unforgeability game and the hash function $H$ from $\mathcal{C}_{h}, \mathcal{T}$ sets $p k_{\mathcal{B}}=(p k, H)$. Whenever $T$ must simulate a request for $\mathcal{E}$ (when receiving (request, $i$ ) from $\mathcal{F}_{\text {OFPOT }}$ or in the complaint buyer phase), $T$ computes values $\left(i, Q_{i}\right)$ and sends $h_{i}=H\left(i, Q_{i}\right)$ to its VES creation oracle to obtain $\omega_{i}$. Upon receiving (complete, $\left.i, 1\right),\left(\operatorname{compSolved}_{\mathcal{V}}\right)$ or $\left(\operatorname{compSolved}_{\mathcal{B}}\right)$ from $\mathcal{F}_{\text {OFPOT }}, \mathcal{T}$ uses its adjudication oracle on input $\omega_{i}$ and $h_{i}=H\left(i, Q_{i}\right)$ to obtain $\sigma_{i}$. Eventually, $\mathcal{E}$ outputs $\left(j, Q_{j}, \omega_{j}\right)$ for new $\left(j, Q_{j}\right)$. $\mathcal{T}$ computes $h_{j}=H\left(j, Q_{j}\right)$. If $h_{j}$ equals any of the previously computed $h_{i}$, $\mathcal{T}$ outputs $\left(\left(j, Q_{j}\right),\left(i, Q_{i}\right)\right)$ in order to break the collision resistance property of $H$. Otherwise $\mathcal{T}$ outputs $\left(\omega_{j}, h_{j}\right)$ as its forgery for the VES scheme. 
Game 4: This game proceeds as Game 3, except that we abort if $\mathcal{E}$ sends a signature $\sigma_{i}$ on a pair $\left(i, Q_{i}\right)$ (or $\left.\left(0, D_{0}\right)\right)$ and $\mathcal{E}$ did not receive before a signature on $\left(i, Q_{i}\right)$. The probability that $\mathcal{Z}$ distinguishes between Game 4 and Game 3 is bounded by the following theorem:

Theorem 9. If the VES scheme is opaque and $H$ is a collision-resistant hash function, $\mid \operatorname{Pr}\left[\right.$ Game 4] $-\operatorname{Pr}\left[\right.$ Game 3] $\mid=\nu_{3}$.

Proof. Given an adversary $\mathcal{E}$ that outputs $\sigma_{i}$ on a new pair $\left(i, Q_{i}\right)$ with non-negligible probability, we construct an algorithm $\mathcal{T}$ that either breaks the collision resistance property of $H$ or wins the opacity game of the VES scheme (see [15]) with non-negligible probability. Upon receiving apk and $p k$ from the challenger $\mathcal{C}_{o}$ of the opacity game and the hash function $H$ from $\mathcal{C}_{h}, \mathcal{T}$ sets $p k_{\mathcal{B}}=(p k, H)$. Whenever $T$ must simulate a request for $\mathcal{E}$ (when receiving (request, $i$ ) from $\mathcal{F}_{\text {OFPOT }}$ or in the complain buyer phase), $T$ computes values $\left(i, Q_{i}\right)$ and sends $h_{i}=H\left(i, Q_{i}\right)$ to its VES creation oracle to obtain $\omega_{i}$. Upon receiving (complete, $\left.i, 1\right),\left(\operatorname{compSolved}_{\mathcal{V}}\right)$ or $\left(\operatorname{compSolved}_{\mathcal{B}}\right)$ from $\mathcal{F}_{\text {OFPOT }}, \mathcal{T}$ uses its adjudication oracle on input $\omega_{i}$ and $h_{i}=H\left(i, Q_{i}\right)$ to obtain $\sigma_{i}$. Eventually, $\mathcal{E}$ outputs $\left(j, Q_{j}, \sigma_{j}\right)$ for new $\left(j, Q_{j}\right) . \mathcal{T}$ computes $h_{j}=H\left(j, Q_{j}\right)$. If $h_{j}$ equals any of the previously computed $h_{i}, \mathcal{T}$ outputs $\left(\left(j, Q_{j}\right),\left(i, Q_{i}\right)\right)$ in order to break the collision resistance property of $H$. Otherwise $\mathcal{T}$ outpus $\left(\sigma_{j}, h_{j}\right)$ to break the opacity property of the VES scheme.

$\mathcal{S}_{\mathcal{V}}$ performs all the changes described in Game 4, and forwards and receives messages from $\mathcal{F}_{\text {OFPOT }}$ as described in our simulation. The distribution produced in Game 4 is identical to that of our simulation. Therefore, by summation we have that $\operatorname{Pr}[$ Game 4$] \leq \nu_{4}$.

Simulation of vendor's security. In this case only the buyer $\mathcal{B}$ is corrupted.

- Upon receiving (crs) from $\mathcal{E}, \mathcal{S}_{\mathcal{B}}$ runs $\mathcal{S}_{\mathcal{B}}^{\prime}$ on input (crs) to get (crs) and sends (crs, crs) to $\mathcal{E}$.

- Upon receiving (init, $\left.p_{1}, \ldots, p_{N}\right)$ from $\mathcal{F}_{\text {OFPOT }}, \mathcal{S}_{\mathcal{B}}$ executes $\mathcal{S}_{\mathcal{B}}^{\prime}$ on input (init, $\left.p_{1}, \ldots, p_{N}\right)$ to obtain $\left(p k_{\mathcal{V}}, T\right)$ and sends them to $\mathcal{E}$.

- Upon receiving $\left(P, p k_{\mathcal{B}}, \sigma_{0}\right)$ from $\mathcal{E}, \mathcal{S}_{\mathcal{B}}$ runs $\mathcal{S}_{\mathcal{B}}^{\prime}$ on input $P$ to obtain $\left(a c_{0}\right.$, $\left.D_{0}\right) . \mathcal{S}_{\mathcal{B}}$ runs OFInitV $\left(p k_{\mathcal{B}}, \sigma_{0}, D_{0}\right)$ and ignores the request if the output is reject. Otherwise $\mathcal{S}_{\mathcal{B}}$ sends (deposit, $\left.a c_{0}\right)$ to $\mathcal{F}_{\text {OFPOT }}$.

- Upon receiving a request from $\mathcal{E}, \mathcal{S}_{\mathcal{B}}$ runs $(a s k, a p k) \leftarrow$ OFInitA $(c r s)$ and sends apk to $\mathcal{E}$.

- Upon receiving $\left(p k_{\mathcal{V}}, p k_{\mathcal{B}}\right)$ from $\mathcal{E}, \mathcal{S}_{\mathcal{B}}$ aborts if they do not equal $p k_{\mathcal{V}}$ previously sent to $\mathcal{E}$ or $p k_{\mathcal{B}}$ previously sent by $\mathcal{E}$.

- Upon receiving $\left(i, Q_{i}, \omega_{i}\right)$ from $\mathcal{E}, \mathcal{S}_{\mathcal{B}}$ runs $\mathcal{S}_{\mathcal{B}}^{\prime}$ on input $Q_{i}$ to obtain $(\tau) . \mathcal{S}_{\mathcal{B}}$ runs OFVerReq $\left(p k_{\mathcal{B}}, a p k, \omega_{i}, i, Q_{i}\right)$ and, if the output is reject, $\mathcal{S}_{\mathcal{B}}$ ignores the request. Otherwise $\mathcal{S}_{\mathcal{B}}$ sends (request, $i, \tau$ ) to $\mathcal{F}_{\text {OFPOT }}$.

- Upon receiving (response, $i, m_{\tau}$ ) from $\mathcal{F}_{\text {OFPOT }}, \mathcal{S}_{\mathcal{B}}$ executes $\mathcal{S}_{\mathcal{B}}^{\prime}$ on input (response, $m_{\tau}$ ) to obtain a response $(R)$ and sends $(R)$ to $\mathcal{E}$.

- Upon receiving $\left(\sigma_{i}\right)$ from $\mathcal{E}, \mathcal{S}_{\mathcal{B}}$ runs $\operatorname{OFVerComp~}\left(p k_{\mathcal{B}}, \sigma_{i}, i, Q_{i}\right)$ and, if the output is reject, sends (complete, $i, 0$ ). Otherwise $\mathcal{S}_{\mathcal{B}}$ sends (complete, $i, 1$ ). 
- Upon receiving $\left(\operatorname{compResp}_{\mathcal{V}}, i, m_{\tau}\right)$ from $\mathcal{F}_{\text {OFPOT }}, \mathcal{S}_{\mathcal{B}}$ executes $\mathcal{S}_{\mathcal{B}}^{\prime}$ on input (response, $m_{\tau}$ ) to obtain a response $(R)$ and sends $(R)$ to $\mathcal{E}$.

- Upon receiving a complain $\left(i, Q_{i}, \omega_{i}\right)$ from $\mathcal{E}, \mathcal{S}_{\mathcal{B}}$ proceeds as for a request to obtain $\tau$ and finally sends ( $\operatorname{complain}_{\mathcal{B}}, i, \tau$ ) to $\mathcal{F}_{\text {OFPOT. }}$.

- Upon receiving $\left(\operatorname{compInv}_{\mathcal{B}}\right)$ from $\mathcal{F}_{\text {OFPOT }}, \mathcal{S}_{\mathcal{B}}$ forwards $\left(\operatorname{compInv}_{\mathcal{B}}\right)$ to $\mathcal{E}$.

- Upon receiving $\left(\operatorname{compResp}_{\mathcal{B}}, i, m_{\tau}\right)$ from $\mathcal{F}_{\text {OFPOT }}, \mathcal{S}_{\mathcal{B}}$ proceeds as for a response and finally sends $R$ to $\mathcal{E}$.

Claim. When only $\mathcal{B}$ is corrupted, the distribution ensembles $\operatorname{IDEAL}_{\mathcal{F}_{\text {OFPOT }}, \mathcal{S}, \mathcal{Z}}$ and $\mathrm{REAL}_{\mathrm{OFPOT}, \mathcal{E}, \mathcal{Z}}$ are computationally indistinguishable under the assumption that vendor's security in the POT scheme holds and under the extractability of the VES scheme.

We show that the real ensemble and the ideal ensemble are indistinguishable.

Game 0: This game corresponds to the execution of the real-world protocol with honest $\mathcal{B}$ and $\mathcal{A}$. Therefore, $\operatorname{Pr}[$ Game 0$]=0$.

Game 1: This game performs all the changes described in $\mathcal{S}_{\mathcal{B}}^{\prime}$. The security proof of the POT scheme demonstrates that $\mid \operatorname{Pr}[$ Game 1] $-\operatorname{Pr}[$ Game 0] $\mid=$ $\nu_{1}$

Game 2: This game proceeds as Game 1, except that the public key apk of the adjudicator is replaced by another valid public key computed via algorithm OFInitA(crs). Since both public keys follow the same distribution $\mid \operatorname{Pr}[$ Game 2] $-\operatorname{Pr}[$ Game 1] $\mid=0$.

$\mathcal{S}_{\mathcal{B}}$ performs all the changes described in Game 2, and forwards and receives messages from $\mathcal{F}_{\text {OFPOT }}$ as described in our simulation. The distribution produced in Game 2 is identical to that of our simulation. Therefore, by summation we have that $\operatorname{Pr}\left[\right.$ Game 2] $\leq \nu_{1}$.

\section{Universally Composable Priced Oblivious Transfer}

We summarize here the POT scheme in [7], modifying the interface offered by the algorithms so that it can be used straightforwardly to instantiate our OFPOT scheme.

\section{C.1 Technical Preliminaries}

Security Assumptions. Let $\left(p, \mathbb{G}, \mathbb{G}_{t}, e, g\right)$ be a pairing group setup. The $l$ Hidden Strong Diffie-Hellman (HSDH) assumption [42] states that, on input $\left(g, g^{\alpha}, u\right) \in \mathbb{G}^{3}$ and a set of tuples $\left(g^{1 /\left(\alpha+c_{i}\right)}, g^{c_{i}}, u^{c_{i}}\right)_{i=1}^{l}$, it is hard to output a new tuple $\left(g^{1 /(\alpha+c)}, g^{c}, u^{c}\right)$. The $l$ Triple Diffie-Hellman (THD) assumption [43] states that, on input $\left(g, g^{x}, g^{y}\right) \in \mathbb{G}^{3}$ and a set of tuples $\left(g^{1 /\left(x+c_{i}\right)}, c_{i}\right)_{i=1}^{l}$, it is hard to output $\left(g^{\mu x}, g^{\mu y}, g^{\mu x y}\right)$ for $\mu \neq 0$. The Decision Linear (DLIN) assumption [44] states that, on input $\left(g, g^{a}, g^{b}, g^{a c}, g^{b d}, z\right) \in \mathbb{G}^{6}$ for random $a, b, c, d \in \mathbb{Z}_{p}$, it is hard to decide whether $z=g^{c+d}$. 
Non-interactive Proofs of Knowledge. Let $R$ be an efficiently computable relation and $L=\{y: \exists w \mid R(y, w)=$ accept $\}$ be an NP-language. For tuples $(y, w) \in R$, we call $y$ the instance and $w$ the witness. A non-interactive proof of knowledge (NIPK) system [45] consists of algorithms (PKSetup, PKProve, PKVerify). Algorithm PKSetup $\left(1^{\kappa}\right)$ outputs a common reference string $\operatorname{par}_{\mathrm{PK}}$. PKProve $\left(\operatorname{par}_{\mathrm{PK}}, y, w\right)$ computes a proof pok of instance $y$ by using witness $w$. PKVerify $\left(\operatorname{par}_{\mathrm{PK}}, y, p o k\right)$ outputs accept if pok is correct. A NIPK system should be complete, sound, and zero-knowledge (or witness-indistinguishable). See [4649] for formal definitions.

A proof of knowledge needs to be extractable, which means that there exists a polynomial time extractor (PKExtSetup, PKExt). PKExtSetup $\left(1^{\kappa}\right)$ generates parameters par $_{\mathrm{PK}}$ that are identically distributed to those generated by PKSetup and an extraction trapdoor $t d_{\text {ext }}$. PKExt $\left(\operatorname{par}_{\mathrm{PK}}, t d_{\text {ext }}, y, p o k\right)$ extracts the witness $w$ with all but negligible probability when $\operatorname{PKVerify}\left(\operatorname{par}_{\mathrm{PK}}, y, p o k\right)$ outputs accept. In an $f$-extractable proof system [43], PKExt extracts a value $z$ such that $\exists w: z=f(w) \wedge(y, w) \in R$. If $f(\cdot)$ is the identity function, we get the usual notion of extractability [45].

We consider NIPK about (unconditionally binding) commitments. ' $x$ in $C$ ' denotes that there exists open such that $C=\operatorname{Com}\left(\operatorname{par}_{\text {com }}, x\right.$, open $)$. The following notation denotes an $f$-extractable NIPK for instance $\left(C_{1}, \ldots, C_{n}\right.$, Condition) with witness $\left(x_{1}\right.$, open $_{1}, \ldots, x_{n}$, open $\left._{n}, s\right)$ that allows extracting all the witness except the openings of the commitments ( $s$ is the part of the witness not related to the commitments in the instance):

$$
\operatorname{NIPK}\left\{\left(x_{1}, \ldots, x_{n}, s\right): \text { Condition }\left(\operatorname{par}_{\mathrm{PK}}, x_{1}, \ldots, x_{n}, s\right) \wedge\left\{x_{i} \text { in } C_{i}\right\}_{i=1}^{n}\right\}
$$

The POT scheme employs the Groth-Sahai NIPK system [50], which allows the computation of witness-indistinguishable proofs on statements about pairing product equations. ${ }^{9}$ The pairing group setup $\left(p, \mathbb{G}, \mathbb{G}_{t}, e, g\right)$ is part of the common reference string $\operatorname{par}_{\mathrm{PK}}$ as output by PKSetup $\left(1^{\kappa}\right)$ and the instance consists of the coefficients $\left\{a_{q}, b_{q}\right\}_{q=1 \ldots Q} \in \mathbb{G}, t \in \mathbb{G}_{t},\left\{\alpha_{q, i}, \beta_{q, i}\right\}_{q=1 \ldots Q, i=1 \ldots m} \in \mathbb{Z}_{p}$ of the pairing product equation: $\prod_{q=1}^{Q} e\left(a_{q} \prod_{i=1}^{m} x_{i}^{\alpha_{q, i}}, b_{q} \prod_{i=1}^{m} x_{i}^{\beta_{q, i}}\right)=t$. The prover knows $\left\{x_{i}\right\}_{i=1}^{m}$ that satisfy this equation.

Internally Groth-Sahai proofs prove relations between commitments. It is possible to add pre-existing Groth-Sahai commitments $\left\{C_{i}\right\}_{i=1 \ldots n}, n \leq m$, to the instance for some of the $x_{i}$ values. The corresponding openings open $n_{i}$ become part of the witness. We can apply the aforementioned notation to Groth-Sahai proofs as follows:

$$
\operatorname{NIPK}\left\{\left(\left\{x_{i}\right\}_{i=1}^{n},\left\{x_{i}\right\}_{i=n+1}^{m}\right): \prod_{q=1}^{Q} e\left(a_{q} \prod_{i=1}^{n} x_{i}^{\alpha_{q, i}}, b_{q} \prod_{i=1}^{m} x_{i}^{\beta_{q, m}}\right)=t \wedge\left\{x_{i} \text { in } C_{i}\right\}_{i=1}^{n}\right\}
$$

We use the instantiation of GS proofs secure under the DLIN assumption.

\footnotetext{
${ }^{9}$ Some classes of equations admit zero-knowledge proofs.
} 
P-Signature Schemes. P-Signatures [43] are signature schemes equipped with a common reference string par $_{\text {sig }}$ and a NIPK that allows proving possession of a signature on a committed message. The POT scheme employs the P-signature scheme (Setup, Kg, Sign, Vf) in [43]. Setup $\left(1^{\kappa}\right)$ runs the GS PKSetup $\left(1^{\kappa}\right)$ to get par $_{\mathrm{PK}}$, picks random $u \in \mathbb{G}$ and outputs par ${ }_{\text {sig }}=\left(\operatorname{par}_{\mathrm{PK}}, u\right) . \mathrm{Kg}\left(\right.$ par $\left._{\text {sig }}\right)$ picks a secret key $s k=(\alpha, \beta) \leftarrow \mathbb{Z}_{p}$ and computes a public key $p k=(v, w)=\left(g^{\alpha}, g^{\beta}\right)$. $\operatorname{Sign}\left(\right.$ par $\left._{\text {sig }}, s k, m\right)$ picks random $r \leftarrow \mathbb{Z}_{p} \backslash\left\{\frac{\alpha-m}{\beta}\right\}$ and outputs $\sigma=\left(\sigma_{1}, \sigma_{2}, \sigma_{3}\right)=$ $\left(g^{1 /(\alpha+m+\beta r)}, w^{r}, u^{r}\right) . \mathrm{Vf}\left(p a r_{s i g}, p k, m, \sigma\right)$ outputs accept if $e\left(\sigma_{1}, v g^{m} \sigma_{2}\right)=e(g$, $g)$ and $e\left(u, \sigma_{2}\right)=e\left(\sigma_{3}, w\right)$. The NIPK is denoted as $\operatorname{NIPK}\left\{\left(g^{m}, u^{m}, \sigma\right): \operatorname{Vf}(p k, \sigma\right.$, $m)=$ accept $\}$. This scheme is $F$-unforgeable ${ }^{10}\left(F(m)=\left(g^{m}, u^{m}\right)\right)$ under the HSDH and THD assumptions.

The POT scheme also employs the P-signature scheme for signing multi-block messages $\left(\operatorname{Setup}_{\mathrm{n}}, \mathrm{Kg}_{\mathrm{n}}, \operatorname{Sign}_{\mathrm{n}}, \mathrm{Vf}_{\mathrm{n}}\right)$ in [7]. Setup $\left(1^{\kappa}\right)$ runs the GS PKSetup $\left(1^{\kappa}\right)$ to get $\operatorname{par}_{\mathrm{PK}}$, picks random $u \in \mathbb{G}$, and outputs par ${ }_{\text {sig }}=\left(\operatorname{par}_{\mathrm{PK}}, u\right) \cdot \mathrm{Kg}_{\mathrm{n}}\left(\right.$ par $\left._{\text {sig }}\right)$ picks random $\left(\alpha, \beta_{1}, \ldots, \beta_{n}, \lambda_{1}, \ldots, \lambda_{n}\right) \leftarrow \mathbb{Z}_{p}$ and sets a public key $P k=$ $\left(v, g_{1}, \ldots, g_{n}, u_{1}, \ldots, u_{n}\right)=\left(g^{\alpha}, g^{\beta_{1}}, \ldots, g^{\beta_{n}}, u^{\lambda_{1}}, \ldots, u^{\lambda_{n}}\right)$ and a secret key $S k=$ $\left(\alpha, \beta_{1}, \ldots, \beta_{n}\right) . \operatorname{Sign}_{\mathrm{n}}\left(\right.$ par $\left._{\text {sig }}, S k, \boldsymbol{m}\right)$ picks random $r \leftarrow \mathbb{Z}_{p} \backslash\left\{-\left(\alpha+\beta_{1} m_{1}+\ldots+\right.\right.$ $\left.\left.\beta_{n} m_{n}\right)\right\}$ and computes a signature $\sigma=\left(\sigma_{1}, \sigma_{2}, \sigma_{3}\right)=\left(g^{1 /\left(\alpha+r+\beta_{1} m_{1}+\ldots+\beta_{n} m_{n}\right)}\right.$, $\left.g^{r}, u^{r}\right)$. Algorithm $\mathrm{Vf}_{\mathrm{n}}\left(\right.$ par $\left._{\text {sig }}, P k, \boldsymbol{m}, \sigma\right)$ outputs accept if $e\left(\sigma_{1}, v \sigma_{2} \prod_{i=1}^{n} g_{i}^{m_{i}}\right)$ $=e(g, g)$ and $e\left(u, \sigma_{2}\right)=e\left(\sigma_{3}, g\right)$. This proof is denoted as NIPK $\left\{\left(\left\{g_{i}^{m_{i}}, u_{i}^{m_{i}}\right\}_{i=1}^{n}\right.\right.$, $\sigma): \mathrm{Vf}_{\mathrm{n}}(P k, \boldsymbol{m}, \sigma)=$ accept $\}$. This scheme is $F$-unforgeable $\left(F(\boldsymbol{m})=\left(g_{1}^{m}, u_{1}^{m}\right.\right.$, $\left.\left.\ldots, g_{n}^{m}, u_{n}^{m}\right)\right)$ under the HSDH and THD assumptions.

We recall that an F-unforgeable signature scheme can also be verified using the $F\left(m_{i}\right)$ values alone. We write, e.g., $\mathrm{Vf}_{\mathrm{n}}\left(P k,\left\langle m_{1}, F\left(m_{2}\right), m_{3}\right\rangle, \sigma\right)$ to indicate that the signature $\sigma$ is verified using only the $F$ value of message $m_{2}$.

Non-Interactive Range Proof. The POT scheme employs the NIPK that a value $\alpha \in \mathbb{Z}_{p}$ lies in an interval $\left[0, A=d^{a}\right.$ ) proposed in [7], which applies to GS proofs the range proof in [27] (see Section 2). It consists of algorithms (InitVer, InitP, RProve). In the initialization phase, verifier runs InitVer $\left(\right.$ par $\left._{\text {sig }}, A\right)$, which, on input par sig output by Setup $\left(1^{\kappa}\right)$, executes $\mathrm{Kg}\left(\right.$ par $\left._{\text {sig }}\right)$ to obtain (sk, $p k)$, computes $A_{i}=\operatorname{Sign}\left(p_{a r} r_{s i g}, s k, i\right)$ for all $i \in \mathbb{Z}_{d}$ and outputs par ran $_{\text {ran }}=$ $\left(p k,\left\{A_{i}\right\}_{i \in \mathbb{Z}_{d}}\right)$, and sends par $_{\text {ran }}$ to the prover. The prover runs InitP $\left(\right.$ par $_{s i g}$, par $\left._{\text {ran }}\right)$, which outputs accept if, for all $i \in \mathbb{Z}_{d}, \operatorname{Vf}\left(\right.$ par $\left._{\text {sig }}, p k, i, A_{i}\right)$ outputs accept. Then the prover can compute multiple proofs. Algorithm RProve $\left(\right.$ par $_{\text {sig }}$, $\operatorname{par}_{\text {ran }}, \tilde{g}, \alpha$, open $\left._{\alpha}\right)$ computes a NIPK that $\alpha$ committed in $C_{\alpha}$ lies in $\left[0, d^{a}\right)$ : $\operatorname{NIPK}\left\{\left(\tilde{g}^{\alpha},\left\{g^{\alpha_{j}}, u^{\alpha_{j}}, A_{\alpha_{j}}\right\}_{j=0}^{a-1}\right):\left\{\mathrm{Vf}\left(p k, \alpha_{j}, A_{\alpha_{j}}\right)\right)\right\}_{j=0}^{a-1} \wedge e\left(g, \tilde{g}^{\alpha}\right) \prod_{j=0}^{a-1} e\left(\tilde{g}^{-d^{j}}\right.$, $\left.g^{\alpha_{j}}\right)=1 \wedge \tilde{g}^{\alpha}$ in $\left.C_{\alpha}\right\}$. This proof is abbreviated as $\operatorname{NIPK}\left\{\left(\tilde{g}^{\alpha}\right): 0 \leq \alpha<\right.$ $A \wedge \tilde{g}^{\alpha}$ in $\left.C_{\alpha}\right\}$.

\section{C.2 Construction}

We recall the algorithms of the POT scheme. They are invoked in the same way we describe for our efficient POT scheme in Section 2.

${ }^{10}$ We refer to [43] for the definition of $F$-unforgeability. 
POTGenCRS $\left(1^{\kappa}, p_{\max }, D_{\max }\right)$ creates two GS reference strings $\operatorname{par}_{\mathrm{PK}}^{\mathcal{V}}$ and $\operatorname{par}_{\mathrm{PK}}^{\mathcal{B}}$ for the same setup $\left(p, \mathbb{G}, \mathbb{G}_{t}, e, g\right)$ ( $\operatorname{such}$ that $\left.-p_{\max }>D_{\max } \bmod p\right)$, computes $\left(h_{1}, h_{2}, h_{3}\right)=\left(g^{a}, g^{b}, g^{c}\right)$ for random $a, b, c \leftarrow \mathbb{Z}_{p}$, picks random $u \leftarrow \mathbb{G}$ and outputs crs $=\left(\operatorname{par}_{\mathrm{PK}}^{\mathcal{V}}, \operatorname{par}_{\mathrm{PK}}^{\mathcal{B}}, u, h_{1}, h_{2}, h_{3}\right) \cdot{ }^{11}$

POTInitV $\left(\right.$ crs $\left., m_{1}, p_{1}, \ldots, m_{N}, p_{N}, D_{\max }\right)$ picks random $x_{1}, x_{2} \leftarrow \mathbb{Z}_{p}$, sets $\left(w_{1}\right.$, $\left.w_{2}\right)=\left(h_{3}^{1 / x_{1}}, h_{3}^{1 / x_{2}}\right)$ and runs $\operatorname{Kg}_{\mathrm{n}}$ to obtain $(P k, S k)$, where $P k=\left(v, g_{1}, g_{2}\right.$, $\left.g_{3}, u_{1}, u_{2}, u_{3}\right)$ and $S k=\left(\alpha, \beta_{1}, \beta_{2}, \beta_{3}\right)$. Then, for $i=1$ to $N$, it picks random $r_{1}, r_{2} \leftarrow \mathbb{Z}_{p}$, computes $\left(\sigma_{1}, \sigma_{2}, \sigma_{3}\right) \leftarrow \operatorname{Sign}_{\mathrm{n}}\left(\operatorname{par}_{\text {sig }}, S k,\left(r_{1}, r_{2}, p_{i}\right)\right)$ and encrypts $m_{i}$ as $C_{i}=\left(w_{1}^{r_{1}}, w_{2}^{r_{2}}, h_{1}^{r_{1}}, h_{2}^{r_{2}}, m_{i} \cdot h_{3}^{r_{1}+r_{2}}, g_{1}^{r_{1}}, g_{2}^{r_{2}}, u_{1}^{r_{1}}, u_{2}^{r_{2}}, \sigma_{1}, \sigma_{2}, \sigma_{3}\right.$, $\left.p_{i}\right)$. It runs InitVer $\left(\right.$ par $\left._{s i g}, D_{\max }\right)$ to obtain $\operatorname{par}_{\text {ran }}$, sets $p k_{\mathcal{V}}=\left(w_{1}, w_{2}, P k\right.$, $\left.\operatorname{par}_{\text {ran }}\right), s k_{\mathcal{V}}=\left(x_{1}, x_{2}\right)$ and $T=\left(C_{1}, \ldots, C_{N}\right)$, and outputs $\left(T, s k_{\mathcal{V}}, p k_{\mathcal{V}}\right)$.

POTInitB $\left(c r s, p k_{\mathcal{V}}, T, a c_{0}\right)$ runs $\operatorname{InitP}\left(\right.$ par $\left._{\text {sig }}, p a r_{\text {ran }}\right)$ to verify $p a r_{\text {ran }}$. For $i=$ 1 to $N$, it runs $\operatorname{Vf}_{\mathrm{n}}\left(P k,\left\langle\left(c_{6}, c_{8}\right),\left(c_{7}, c_{9}\right), p_{i}\right\rangle, \sigma\right)$, where $\sigma=\left(\sigma_{1}, \sigma_{2}, \sigma_{3}\right)$. It checks that $e\left(c_{1}, h_{1}\right)=e\left(c_{3}, w_{1}\right), e\left(c_{2}, h_{2}\right)=e\left(c_{4}, w_{2}\right), e\left(h_{1}, c_{6}\right)=e\left(c_{3}, g_{1}\right)$, $e\left(h_{2}, c_{7}\right)=e\left(c_{4}, g_{2}\right)$. If all these checks hold, it picks random $\left(l_{1}, l_{2}\right) \leftarrow$ $\mathbb{Z}_{p}$, runs and encrypts $c t=\left(c_{1}, c_{2}, c_{3}\right)=\left(w_{1}^{l_{1}}, w_{2}^{l_{2}}, a c_{0} \cdot h_{3}^{l_{1}+l_{2}}\right)$. It picks random open ${ }_{a c_{0}}$, computes $D_{0}=\operatorname{Com}\left(\operatorname{par}_{\mathrm{PK}}^{\mathcal{B}}, g_{3}^{a c_{0}}\right.$ open $\left._{a c_{0}}\right)$ and outputs $P=\left(\right.$ ct, open $\left.{ }_{a c_{0}}\right), D_{0}^{\prime}=\left(a_{0}\right.$, open $\left._{a c_{0}}, D_{0}\right) .{ }^{12}$ If not, it outputs reject.

POTGetDep $\left(c r s, s k_{\mathcal{V}}, P, D_{\max }\right)$ computes $a c_{0}=c_{3} /\left(c_{1}^{x_{1}} c_{2}^{x_{2}}\right)$, checks that $a c_{0} \in$ $[0, A)$ and computes $D_{0}=\operatorname{Com}\left(\operatorname{par}_{\mathrm{PK}}^{\mathcal{B}}, g_{3}^{a c_{0}}\right.$, open $\left._{a c_{0}}\right)$. It outputs $\left(D_{0}, a c_{0}\right)$.

$\operatorname{POTReq}\left(c r s, p k_{\mathcal{V}}, T, D_{i-1}^{\prime}, \tau\right)$ parses $C_{\tau}$ as $\left(c_{1}, c_{2}, c_{3}, c_{4}, c_{5}, c_{6}, c_{7}, c_{8}, c_{9}, \sigma_{1}, \sigma_{2}\right.$, $\left.\sigma_{3}, p_{\tau}\right)$. It picks random $y_{1}, y_{2} \leftarrow \mathbb{Z}_{p}$ and computes $\left(d_{1}, d_{2}\right)=\left(c_{1} \cdot w_{1}^{y_{1}}, c_{2} \cdot w_{2}^{y_{2}}\right)$ and $\left(t_{1}, t_{2}\right)=\left(h_{3}^{y_{1}}, h_{3}^{y_{2}}\right)$. It also picks a fresh open ${ }_{a c_{i}}$ to compute $D_{i}=$ $\operatorname{Com}\left(g_{3}^{a c_{i}}\right.$, open $\left._{a c_{i}}\right)$, for $a c_{i}=a c_{i-1}-p_{\tau}$. It runs PKProve on input par ${ }_{\mathrm{PK}}^{\mathcal{B}}$ to compute a witness-indistinguishable proof $p o k_{1}$ :

$$
\begin{aligned}
\mathrm{NIPK} & \left\{\left(c_{6}, c_{8}, c_{7}, c_{9}, g_{3}^{p_{\tau}}, u_{3}^{p_{\tau}}, \sigma_{1}, \sigma_{2}, \sigma_{3}, g_{3}^{a c_{i}}, g_{3}^{a c_{i-1}}, c_{1}, c_{2}, t_{1}, t_{2}\right):\right. \\
& \mathrm{Vf}_{\mathrm{n}}\left(P k,\left\langle\left(c_{6}, c_{8}\right),\left(c_{7}, c_{9}\right),\left(g_{3}^{p_{\tau}}, u_{3}^{p_{\tau}}\right)\right\rangle,\left(\sigma_{1}, \sigma_{2}, \sigma_{3}\right)\right)=\text { accept} \wedge \\
& e\left(w_{1}^{-1}, c_{6}\right) e\left(c_{1}, g_{1}\right)=1 \wedge e\left(w_{2}^{-1}, c_{7}\right) e\left(c_{2}, g_{2}\right)=1 \wedge \\
& e\left(c_{1}, h_{3}\right) e\left(t_{1}, w_{1}\right)=e\left(d_{1}, h_{3}\right) \wedge e\left(c_{2}, h_{3}\right) e\left(t_{2}, w_{2}\right)=e\left(d_{2}, h_{3}\right) \wedge \\
& e\left(g, g_{3}^{a c_{i-1}}\right) e\left(g^{-1}, g_{3}^{a c_{i}}\right) e\left(g^{-1}, g_{3}^{p_{\tau}}\right)=1 \wedge \\
& \left.\wedge 0 \leq a c_{i}<A \wedge g_{3}^{a c_{i}} \text { in } D_{i} \wedge g_{3}^{a c_{i-1}} \text { in } D_{i-1}\right\}
\end{aligned}
$$

It outputs $Q=\left(d_{1}, d_{2}\right.$, pok $\left._{1}, D_{i}\right), Q^{\prime}=\left(Q, \tau, y_{1}, y_{2}\right)$ and $D_{i}^{\prime}=\left(a c_{i}\right.$, open $_{a c_{i}}$, $\left.D_{i}\right)$.

POTVerReq $\left(c r s, p k_{\mathcal{V}}, D_{i-1}, Q\right)$ verifies $p o k_{1}$ by running PKVerify on input par ${ }_{\mathrm{PK}}^{\mathcal{B}}$ and it outputs reject if it fails. For this verification, it uses the commitments $D_{i-1}$ and $D_{i}$.

${ }^{11} \operatorname{par}_{\text {sig }}$ is $\left(\operatorname{par}_{\mathrm{PK}}^{\mathcal{V}}, u\right)$ and is used for both the single message and multi-block message P-signature schemes.

12 The original description uses open ${ }_{a c_{0}}=0$. We take a random value because these allows us to hide $a c_{0}$ from the adjudicator. 
POTResp $\left(c r s, p k_{\mathcal{V}}, s k_{\mathcal{V}}, Q\right)$ computes $\left(z_{1}, z_{2}\right)=\left(d_{1}^{x_{1}}, d_{2}^{x_{2}}\right)$ and $z=z_{1} \cdot z_{2}$ and runs PKProve on input $\operatorname{par}_{\mathrm{PK}}^{\mathcal{V}}$ to get a zero-knowledge proof of knowledge ${ }^{13}$ pok $_{2}$ :

$$
\begin{gathered}
\operatorname{NIPK}\left\{\left(z_{1}, z_{2}\right): e\left(z_{1}, w_{1}\right)=e\left(d_{1}, h_{3}\right) \wedge e\left(z_{2}, w_{2}\right)=e\left(d_{2}, h_{3}\right)\right. \\
\left.\wedge e\left(z_{1}, h_{3}\right) e\left(z_{2}, h_{3}\right)=e\left(z, h_{3}\right)\right\}
\end{gathered}
$$

It outputs $R=\left(z, p o k_{2}\right)$.

POTVerResp $\left(c r s, p k_{\mathcal{V}}, R\right)$ verifies $p_{2} k_{2}$ by running PKVerify on input $\operatorname{par}_{\mathrm{PK}}^{\mathcal{V}}$. If verification fails, it outputs reject.

POTComplete $\left(\mathrm{crs}, T, R, Q^{\prime}\right)$ parses $C_{\tau}$ to obtain $c_{5}$ and outputs the message $m_{\tau}=c_{5} /\left(z \cdot h_{3}^{-y_{1}} \cdot h_{3}^{-y_{2}}\right)$.

\section{C.3 Security Proof}

Theorem 10. This POT scheme securely realizes $\mathcal{F}_{P O T}$ under the DL, HSDH and THD assumptions.

We depict here the simulator $\mathcal{S}^{\prime}=\left\{\mathcal{S}_{\mathcal{V}}^{\prime}, \mathcal{S}_{\mathcal{B}}^{\prime}\right\}$ given by [7] since it is used by the simulator $\mathcal{S}$ in the security proof of the OFPOT scheme, but we refer to [7] for the rest of the proof.

In the proof it is used the fact that Groth-Sahai proofs are partially extractable, composable witness-indistinguishable, and (given certain conditions) composable zero-knowledge. Algorithms (PKSimSetup, PKSimProve) are utilized to simulate proofs. PKSimSetup $\left(1^{\kappa}\right)$ outputs an alternative setup par $_{\mathrm{PK}}^{\prime}$ and a simulation trapdoor $t d_{\text {sim }}$. PKSimProve $\left(\operatorname{par}_{\mathrm{PK}}^{\prime}, t d_{\text {sim }}, y\right)$, on input simulation parameters $\operatorname{par}_{\mathrm{PK}}^{\prime}$, outputs a proof pok for instance $y$ such that PKVerify $\left(\right.$ par $_{\mathrm{PK}}^{\prime}$, $y$, pok) outputs accept.

Simulation of buyer's security. In this case only the vendor $\mathcal{V}$ is corrupted.

- $\mathcal{S}_{\mathcal{V}}^{\prime}$ runs PKSetup to generate two Groth-Sahai reference strings $\operatorname{par}_{\mathrm{PK}}^{\mathcal{B}}$ and $\operatorname{par}_{\mathrm{PK}}^{\mathcal{V}}$ for the same pairing group setup $\left(p, \mathbb{G}, \mathbb{G}_{t}, e, g\right)$, where $-p_{\max }>D_{\max }$ $\bmod p$ holds. $\mathcal{S}_{\mathcal{V}}^{\prime}$ picks random $u \leftarrow \mathbb{G}$ and $a, b, c \leftarrow \mathbb{Z}_{p}$ and computes $h_{3}=$ $g^{c}$ and $\left(h_{1}, h_{2}\right)=\left(h_{3}^{1 / a}, h_{3}^{1 / b}\right) . \mathcal{S}_{\mathcal{V}}^{\prime}$ sets crs $=\left(\operatorname{par}_{\mathrm{PK}}^{\mathcal{V}}, \operatorname{par}_{\mathrm{PK}}^{\mathcal{B}}, u, h_{1}, h_{2}, h_{3}\right)$. When $\mathcal{F}_{\mathrm{CRS}}$ is queried, $\mathcal{S}_{\mathcal{V}}^{\prime}$ returns (crs).

- Upon receiving $\left(p k_{\mathcal{V}}, T\right)$ from $\mathcal{E}, \mathcal{S}_{\mathcal{V}}^{\prime}$ checks $\left(p k_{\mathcal{V}}, T\right)$ as depicted in POTInitB and aborts if it is not correct. Otherwise $\mathcal{S}_{\mathcal{V}}^{\prime}$ parses $T$ as $\left(C_{1}, \ldots, C_{N}\right)$. For $i=1, \ldots, N, \mathcal{S}_{\mathcal{V}}^{\prime}$ parses $C_{i}$ to get $\left(c_{3}, c_{4}, c_{5}, p_{i}\right)$ and sets $m_{i}=c_{5} /\left(c_{3}^{a} c_{4}^{b}\right) . \mathcal{S}_{\mathcal{V}}^{\prime}$ sends (init, $\left.m_{1}, p_{1}, \ldots, m_{N}, p_{N}\right)$ to $\mathcal{F}_{\text {POT. }}$.

- Upon receiving (deposit, $\left.a c_{0}\right)$ from $\mathcal{F}_{\mathrm{POT}}, \mathcal{S}_{\mathcal{V}}^{\prime}$ computes $\left(P, D_{0}^{\prime}\right)$ as explained in POTInitB. $\mathcal{S}_{\mathcal{V}}^{\prime}$ sends $(P)$ to $\mathcal{E}$ and keeps $D_{0}^{\prime}$.

13 To let this proof be zero-knowledge a new variable $z_{3}$ is introduced. The set of equations is $e\left(z_{1}, w_{1}\right) e\left(d_{1}^{-1}, z_{3}\right)=1 \wedge e\left(z_{2}, w_{2}\right) e\left(d_{2}^{-1}, z_{3}\right)=1 \wedge e\left(z_{1} z_{2}, z_{3}\right) e\left(z^{-1}, z_{3}\right)=$ $1 \wedge e\left(w_{1}, z_{3}\right)=e\left(w_{1}, h_{3}\right)$. 
- In the $i$ th transfer phase, upon receiving (request) from $\mathcal{F}_{\mathrm{POT}}, \mathcal{S}_{\mathcal{V}}^{\prime}$ executes POTReq $\left(c r s, p k_{\mathcal{V}}, T, D_{i-1}^{\prime}, \tau_{\text {min }}\right)$, where $\tau_{\min }$ corresponds to the message with the lowest price, to obtain $\left(Q, Q^{\prime}, D_{i}^{\prime}\right)$, and sends $Q$ to $\mathcal{E}$.

- Upon receiving the response $R$ from $\mathcal{E}, \mathcal{S}_{\mathcal{V}}^{\prime}$ runs POTVerResp $\left(c r s, p k_{\mathcal{V}}, R\right)$. If the output is reject, $\mathcal{S}_{\mathcal{V}}^{\prime}$ sends (response, 0 ) to $\mathcal{F}_{\text {POT }}$. Otherwise, $\mathcal{S}_{\mathcal{V}}^{\prime}$ sends (response, 1) to $\mathcal{F}_{\mathrm{POT}}$ and keeps $D_{i}^{\prime}$.

Simulation of vendor's security. In this case only the buyer $\mathcal{B}$ is corrupted.

- $\mathcal{S}_{\mathcal{B}}^{\prime}$ runs PKExtSetup to obtain $\operatorname{par}_{\mathrm{PK}}^{\mathcal{B}}$ and an extraction trapdoor $t d_{\text {ext }}$, and algorithm PKSimSetup to obtain $\operatorname{par}_{\mathrm{PK}}^{\mathcal{V}}$ and a simulation trapdoor $t d_{s i m}$. Both use the same pairing group setup $\left(p, \mathbb{G}, \mathbb{G}_{t}, e, g\right)$, where $-p_{\max }>D_{\max }$ $\bmod p$ holds. $\mathcal{S}_{\mathcal{B}}^{\prime}$ picks random $a, b, c \leftarrow \mathbb{Z}_{p}$ and computes $\left(h_{1}, h_{2}, h_{3}\right)=$ $\left(g^{a}, g^{b}, g^{c}\right) . \mathcal{S}_{\mathcal{B}}^{\prime}$ picks random $u \leftarrow \mathbb{G}$ and sets crs $=\left(\operatorname{par}_{\mathrm{PK}}^{\mathcal{V}}, \operatorname{par}_{\mathrm{PK}}^{\mathcal{B}}, u, h_{1}, h_{2}\right.$, $\left.h_{3}\right)$. $\mathcal{S}_{\mathcal{B}}^{\prime}$ returns (crs) when $\mathcal{F}_{\mathrm{CRS}}$ is queried.

- Upon receiving (init, $p_{1}, \ldots, p_{N}$ ) from $\mathcal{F}_{\mathrm{POT}}, \mathcal{S}_{\mathcal{B}}^{\prime}$ picks random messages $m_{1}^{\prime}$, $\ldots, m_{N}^{\prime} \in \mathbb{G}_{t}^{N}$ and runs POTInitV $\left(c r s, m_{1}^{\prime}, p_{1}, \ldots, m_{N}^{\prime}, p_{N}, D_{\text {max }}\right)$ to obtain $\left(p k_{\mathcal{V}}, T\right) . \mathcal{S}_{\mathcal{B}}^{\prime}$ sends $\left(p k_{\mathcal{V}}, T\right)$ to $\mathcal{E}$.

- Upon receiving $(P), \mathcal{S}_{\mathcal{B}}^{\prime}$ runs $\left(D_{0}, a c_{0}\right) \leftarrow \operatorname{POTGetDep}\left(c r s, s k_{\mathcal{V}}, P, D_{\max }\right)$, sends (deposit, $a c_{0}$ ) to $\mathcal{F}_{\mathrm{POT}}$ and keeps $D_{0}$.

- In the $i$ th transfer phase, upon receiving $(Q)$ from $\mathcal{E}, \mathcal{S}_{\mathcal{B}}^{\prime}$ parses $Q$ as $\left(d_{1}, d_{2}, p o k_{1}, D_{i}\right) . \mathcal{S}_{\mathcal{B}}^{\prime}$ verifies the proof pok $k_{1}$ by running PKVerify and utilizing $\left(D_{i}, D_{i-1}\right)$, and aborts if verification fails. Otherwise, $\mathcal{S}_{\mathcal{B}}^{\prime}$ executes PKExt $\left(\right.$ par $_{\mathrm{PK}}^{\mathcal{B}}, t d_{\text {ext }}$, pok $\left._{1}\right)$ to extract the witness. Then, for $i=1$ to $N, \mathcal{S}_{\mathcal{B}}^{\prime}$ compares the signature $\left(g_{1}^{r_{1}}, u_{1}^{r_{1}}, g_{2}^{r_{2}}, u_{2}^{r_{2}}, g_{3}^{p_{i}}, u_{3}^{p_{i}}, \sigma_{1}, \sigma_{2}, \sigma_{3}\right)$ in the witness with each of the signatures that are included in the ciphertexts that were sent to $\mathcal{E}$ in order to know the choice $\tau_{i}$ selected by $\mathcal{E}$. $\mathcal{S}_{\mathcal{B}}^{\prime}$ also compares the signatures $\left\{g^{\sigma_{j}}, u^{\sigma_{j}}, A_{\sigma_{j}}\right\}_{j=0}^{a-1}$ in the witness that correspond to the range proof with each of the signatures that were sent to $\mathcal{E}$ in par $_{\text {ran }}$. (This is done in order to ensure that $\mathcal{E}$ did not compute a forgery.) $\mathcal{S}_{\mathcal{B}}^{\prime}$ stores $D_{i}$ and sends (request, $\tau_{i}$ ) to $\mathcal{F}_{\mathrm{POT}}$.

- $\mathcal{S}_{\mathcal{B}}^{\prime}$ receives either (response, $m_{\tau_{i}}$ ) from $\mathcal{F}_{\mathrm{POT}}$. If $m_{\tau_{i}}=\perp, \mathcal{S}_{\mathcal{B}}^{\prime}$ sends $(\perp$ ) to $\mathcal{E}$. Otherwise $\mathcal{S}_{\mathcal{B}}^{\prime}$ uses the value of the ciphertext $c_{5}=m_{i}^{\prime} \cdot h_{3}^{r_{1}+r_{2}}$ and the values $\left(t_{1}, t_{2}\right)=\left(h_{3}^{y_{1}}, h_{3}^{y_{2}}\right)$ in the extracted witness to compute a response $z=\left(c_{5} t_{1} t_{2}\right) / m_{\tau_{i}}$, and uses trapdoor $t d_{s i m}$ to simulate proof $p o k_{2}$. $\mathcal{S}_{\mathcal{B}}^{\prime}$ sets $R=\left(z, p_{0} k_{2}\right)$ and sends $(R)$ to $\mathcal{E}$. 